\title{
Lazaroa
}

ISSN-e 1988-3307

\section{Bioindicators and bioclimatic data as essential tools towards a consistent biogeographic district typology of Sierra Nevada National Park (Spain)}

\author{
José Miguel Marfil ${ }^{1}$ Joaquín Moleroํㅜ Paloma Cantó $\&$ Salvador Rivas-Martínez ${ }^{3}$
}

Received 22 February 2016 / Accepted 22 March 2016

\begin{abstract}
The relationship, through the application of robust and solvent bioclimatic indexes, between the characteristics of the natural environment (geology, orography, climate, etc.) and the recognition of species, communities, and vegetation series, has made it possible to establish in the Sierra Nevada National Park (Spain) a total of 8 homogeneous spaces (districts), included in other larger spaces ( 5 sectors, 2 provinces), which constitute the biogeographic typology of the territory. The consideration, of historical climatic changes and human action, among other factors, shows the necessary use of phytosociological methodology in the characterization of species, communities, and vegetation series. The application of the Aridic Index and the positive temperature, in the bioclimatic context, explains the generalized dryness in the territory and the apparent contradiction of spaces with dry and semiarid ombroclimate in the highest summits. The recognition of homogeneous territories (districts), is an essential tool for the management of this national park.
\end{abstract}

Keywords: Bioclimatology; Aridic Index; Biogeography; Districts; Frigid Territory.

\section{[es] Bioindicadores y datos bioclimáticos como herramientas esenciales para una tipología biogeográfica distrital consistente en el Parque Nacional de Sierra Nevada (España)}

Resumen. La relación, a través de la aplicación de índices bioclimáticos robustos y solventes, entre las características del entorno natural (geología, orografía, clima, etc.) y el reconocimiento de especies, comunidades y series de vegetación, ha permitido establecer en el Parque Nacional de Sierra Nevada (España) un total de 8 espacios homogéneos (distritos), incluidos en otros espacios más amplios (5 sectores, 2 provincias), que constituyen la tipología biogeográfica del territorio. La consideración, entre otros factores, de los cambios climáticos históricos y de la acción humana, demuestra el uso necesario de la metodología fitosociológica en la caracterización de especies, comunidades y series de vegetación. La aplicación del Índice Arídico y la temperatura positiva, en el contexto bioclimático, explica la aridez generalizada en el territorio y la aparente contradicción de los espacios con ombroclima seco y semiárido en las cumbres más altas. El reconocimiento de territorios homogéneos (distritos), es una herramienta esencial para la gestión de este parque nacional.

Palabras clave: Bioclimatología; Índice de Aridez; Biogeografía; Distritos; Territorio Frío.

\section{Introduction}

Sierra Nevada (Cordillera Penibética) is a mountain range in the south-eastern Iberian Peninsula. In the centre lies Sierra Nevada National Park (created on 1999), which has the highest summits of the range (Mulhacén $3479 \mathrm{~m}$ asl, Veleta $3396 \mathrm{~m}$ asl, Alcazaba 3366 $\mathrm{m}$ asl, etc.). It occupies 85883 ha surrounded by the Peripheral Protection Zone of Natural Park of 86355 ha. Located between $37^{\circ} 15^{\prime} \mathrm{N}$ (Lugros) and 36 $55^{\circ} \mathrm{S}$ (Lanjarón) and between

\footnotetext{
Department of Botany, University of Granada, Granada, Spain. Email: jmolero@ugr.es.

Department of Plant Biology II, University Complutense, E-28040 Madrid, Spain.

Phytosociological Research Center, Collado-Villaba, Madrid, Spain.
} 
$3^{\circ} 38^{\prime} \mathrm{W}$ (Suspiro del Moro) and $2^{\circ} 35^{\prime} \mathrm{E}$ (Terque), the total preserve area of Sierra Nevada National Park covers 172238 ha coinciding with the UNESCO Biosphere Reserve Zone (1986). Its elevation ranges between $300 \mathrm{~m}$ asl at the Andarax river near Terque to $3479 \mathrm{~m}$ on Mulhacén peak. The main rivers having their source in Sierra Nevada National Park are: the Genil, Nacimiento, Andarax, Grande de Adra, and Guadalfeo.

According to Martín \& al. (2008), the Penibetic Range and especially its core, Sierra Nevada, belong mainly to the Nevado-Filabride geological complex. It is formed by several overlapping tectonic units ( $>250 \mathrm{Myr})$ : graphitoids and dark micaschists, prevail in the lower one, called "Veleta", while clear micaschists predominate in the upper one, called "Mulhacén". All of these are mixed with gneissic igneous acidic and, above all, by the basic ultramafic rocks (Vera \& al., 2004). The Triassic Alpujarride complex (210-240 Myr), called El Calar, is located above and around the central core, in which marbles, limestones, and dolomites are common, as well as phyllites or launas at the base.

Numerous studies on the structure, composition, and distribution of plant communities have been conducted in the national park and its environment according to the phytosociological and geobotanical Braun-Blanquet method (Géhu \& Rivas-Martínez, 1981; Capelo, 2003; Géhu, 2006). These include Quézel, 1953; Rivas-Martínez, 1961; Rivas Goday \& Ri-
vas-Martínez, 1968, 1971; Prieto, 1971; Martínez-Parras \& Molero Mesa, 1983a, b; Valle, 1985, Losa Quintana \& al., 1986; Rivas-Martínez \& al., 1986; Martínez-Parras \& al., 1987a, b, c; Molero Mesa \& Pérez Raya, 1987a, b; Mota \& Valle, 1987; Pérez Raya, 1987; Pérez Raya \& Molero Mesa, 1988a, b, 1989; Lorite \& al., 1997, 2003, 2007; Molero Mesa 1999; Molero Mesa \& al., 2001; Salazar \& al., 2001; Mota \& al., 2002; Fernández Calzado, 2007; Fernández Calzado \& Molero Mesa, 2011a, b. Marfil (2017) offers an overview and includes a total of 205 plant associations, 118 alliances, and 73 orders. The syntheses of Rivas-Martínez \& al. (2001, 2002a, b, 2011a, b), which include an exhaustive text and checklist with the description and references of all phytosociological communities known in Spain and Portugal. These papers, are the bases for arranging the hierarchical communities of the Sierra Nevada National Park.

Pollen analyses made in the Padul peatlands indicate the alternation of cold and warm-humid climatic periods in the Sierra Nevada between 46 and $4.5 \mathrm{ka}$ (Flörstchütz \& al., 1971; Pons \& Reille, 1988). Glacial events of the Holocene show a variable climatic history on the mountain. According to several authors (Gómez Ortiz \& al., 2002, 2012; Anderson \& al., 2011; Oliva \& al., 2010, 2011, 2014; Jiménez-Moreno \& Anderson, 2012; Oliva \& Gómez Ortiz, 2012, Jiménez-Moreno \& al., 2013; and Jiménez-Moreno, 2016), the main glacier, climatic, and anthropogenic events can be summarized in Table 1 .

Table 1. Main glacial and interglacial periods in Sierra Nevada since last Würm

\begin{tabular}{|l|l|}
\hline 32-30Ka - Wurm maximum & Sierra Nevada glacial maximum \\
\hline $15-14 \mathrm{Ka}$ - Late glacial. Glaciers melting & Glaciers restricted to summits areas \\
\hline $11-7 \mathrm{Ka}$ - Old Holocene & Complete glacier melts; temperature, precipitation increase \\
\hline $7-4 \mathrm{Ka}$ - Middle Holocene. Climatic optimum & Higher temperature and rainfalls \\
\hline 4-2.3 $\mathrm{Ka}$ - Anthropic impact begins & Alternation of aridic to rainy and cold to warmer periods \\
\hline $2.3-0.85 \mathrm{Ka}-$ Roman and Medieval periods & Precipitation increases moderately \\
\hline $0.85-0.15 \mathrm{Ka}$ - Little Ice Age & Temp. decreases $\geq 1.0^{\circ} \mathrm{C}$. Snowfields and glaciers in summits \\
\hline 21 th century & One small buried rock glacier remain in "Corral del Veleta" \\
\hline
\end{tabular}

\section{Material and Methods}

\section{Bioclimatology}

Bioclimatology, a contemporaneous geobotanical science, studies the reciprocity between the climate and the distribution of living beings, mostly plants and their communities on Earth. The increasingly detailed data on the distribution of vegetation, as well as shifts in the appearance and composition of the natural potential vegetation and its substitution stages, 
caused by climatic, edaphic, geographic and anthropogenic factors, are making easier to recognize the bioclimates and the natural potential vegetation frontiers with steadily greater precision and objectivity. Once the bounds of the vegetation series (sigmetum), geoseries (geosigmetum), permaseries (permasigmetum) and geopermaseries (geopermasigmetum) are known, as well as the bioindicators (i.e. communities, soils, and plant species), it will be possible to calculate the numerical bioclimatic threshold values that distinguish them. These spaces corresponding to the bioclimatic units (bioclimates, thermotypes, ombrotypes, continentality, and isobioclimates) have been progressively delimited and adjusted. Numerous investigations have been performed in this respect (Rivas-Martínez, 1976, 1981, 1982a, b, 1983, 1984, 1988, 1991, 1994, 1996, 2004, 2005b; Loidi, 1991; Rivas-Martínez \& al., 1991b, 2011c; Peinado \& al., 1992; Sánchez Gómez \& al., 1993, 1994; Del Arco \& al., 1996, 2002; Rivas-Martínez \& Costa 1998; Lousã, 2004; Mesquita \& al., 2004; Gehú, 2006; Cantó, 2007). The synoptic table "Worldwide bioclimatic classification system", has been recently updated [13.02.2017] by Rivas-Martínez, Rivas-Sáenz \& A. Penas (Table 2).

Table 3 displays the information from 34 meteorological stations inside or surrounding Sierra Nevada National Park. These data help to establish the bioclimates within the Mediterranean macrobioclimate at each station (pluviseasonal oceanic: mepo; xeric oceanic: mexo; desertic oceanic: medo), as well as the thermo-ombroclimatic types (isobioclimates) and to deduce the different indices that show other bioclimatic characters. The Sierra Nevada Global Change Observatory (Environmental and Regional Planning Council of the Regional Government of Andalusia-Spanish National Park Service) is currently monitoring the climatic conditions over the last 50 years (Perez-Luque \& al., 2016a, b; Aspizua \& al., 2012), taking into account numerous other parameters (Zamora \& al., 2016), the results of which reveal possible climatic changes and help guide management for the conservation of the park and its sustainable future.

With the vertical zonation of vegetation series, thermotypes, ombrotypes, and altitudinal bioclimatic belts, we can also recognize and define the territory on the basis of bioclimatic indexes, such as: Thermicity Index (It $=$ $10(\mathrm{~T}+\mathrm{m}+\mathrm{M}) ; \mathrm{m}=$ mean minimum temperature of the coldest month; $\mathrm{M}=$ mean maximum temperature of the coldest month of the year); Annual Positive Temperature ( $\mathrm{Tp}=$ sum of monthly mean temperature higher than $\left.0^{\circ} \mathrm{C}\right)$; Annual Positive Precipitation $(\mathrm{Pp}=$ sum of monthly rainfall for those months with mean temperature $>0^{\circ} \mathrm{C}$ ); Ombrothermic Index $(\mathrm{Io}=10(\mathrm{Pp} / \mathrm{Tp}))$; Continentality Index (Ic: Annual monthly thermic interval $=$ Tmax-Tmin); and Aridic Index (AI), which numerically expresses the aridity value lower than $I o=2(A I Y=$ Annual Aridic Index $=200$ $\left.100\left(\operatorname{Io}_{1+2+\ldots .12}\right)\right)$.

An initial approach to the isobioclimas (thermo- and ombrotypes), bioclimatic belts and application of the Aridic Index was found in Molero \& Marfil (2015). The use of thermometric data provided by Target Region 2 in the Sierra Nevada, within the GLORIA project (Pauli \& al., 2012; Gottfried \& al., 2012; Fernández-Calzado \& al., 2012; Winkler \& al., 2016), has been useful in confirming the data of the cryoromediterranean summits, and extrapolated from the meteorological stations.

\section{Biogeography}

Biogeography is a terrestrial science concerning the distribution of species, plant communities, habitats, biocoenosis, ecosystems, biomes and bioregions on Earth, as well as the relationships between them and their conditions. It takes into account the areas of taxa and syntaxa (chorology), in addition to information from other natural sciences (Geography, Botany, Synecology, Soil Science, Bioclimatology, Geology, etc.), and attempts to establish a hierarchic biogeographic typology of the lands on Earth. The main systematic unit ranks from the higher to lower are: kingdom, region, province, sector, district, country, landscape cell, tesela, and permatesela (Rivas-Martínez \& al., 2007, 2011b, 2014). Terrestrial biogeography has been twinned with phytogeography due to the value and information of the plant species and their communities as bioindicators, in the definition and delimitation of its units. 


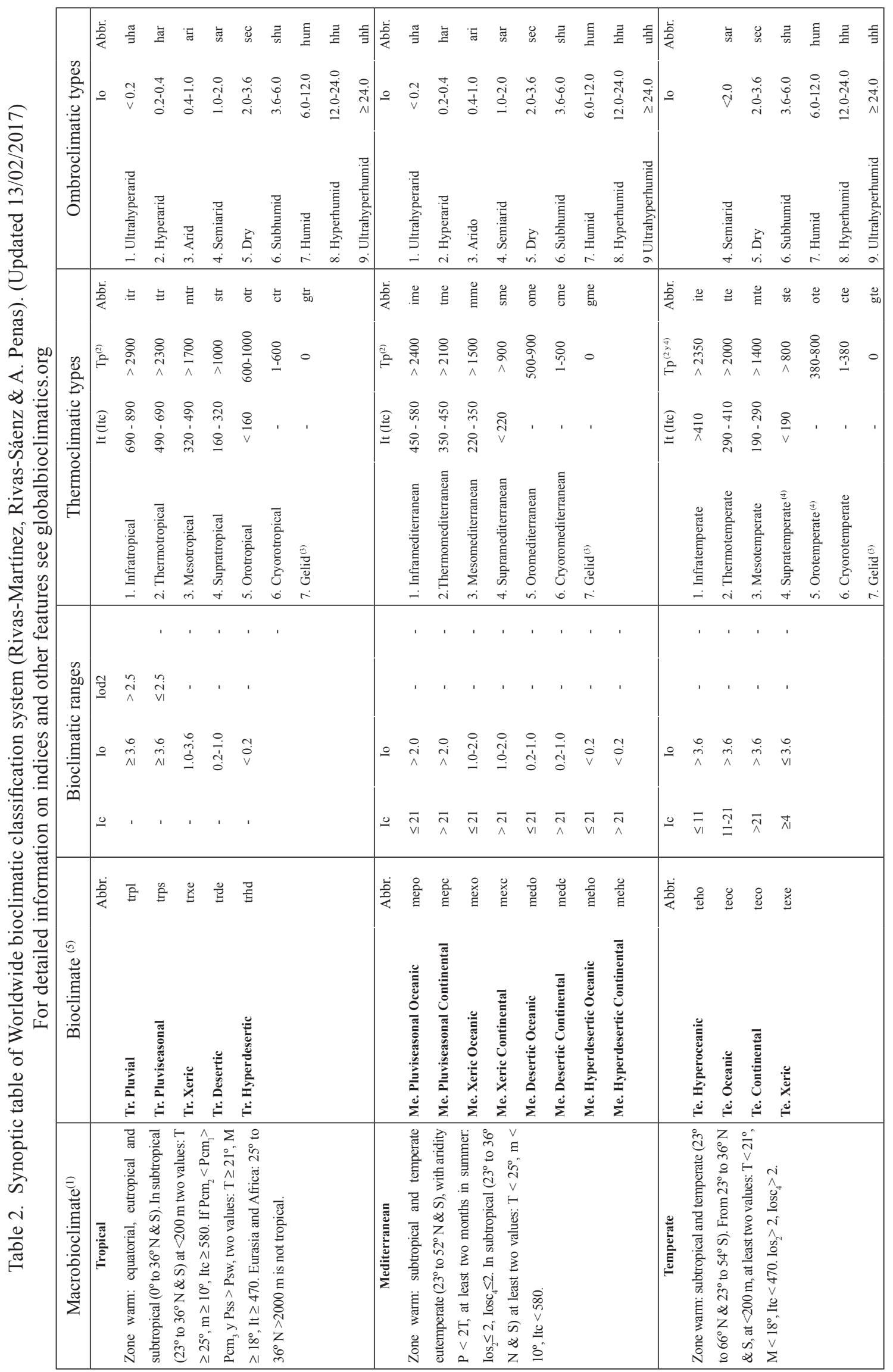




\begin{tabular}{|c|c|c|c|c|c|c|c|c|c|c|c|c|c|c|}
\hline 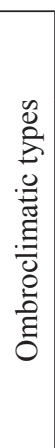 & 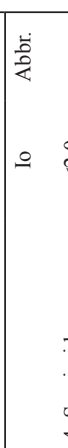 & 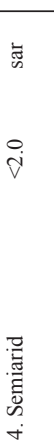 & $\begin{array}{l}\mathscr{ष} \\
\\
0 \\
\dot{\leftrightarrow} \\
\dot{d} \\
\dot{i}\end{array}$ & 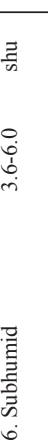 & & & & 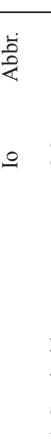 & & $\begin{array}{c}\text { D. } \\
\text { in } \\
\text { in }\end{array}$ & 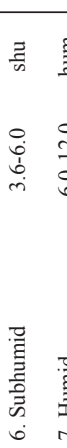 & $\begin{array}{ll} \\
\\
\end{array}$ & 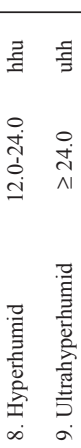 & 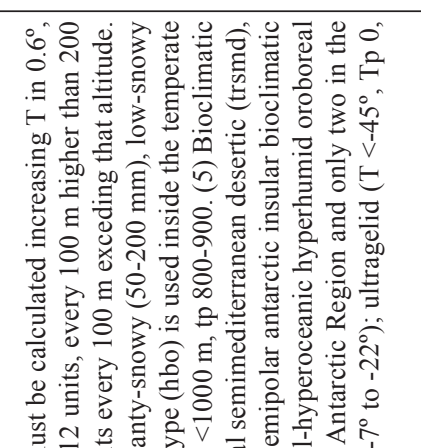 \\
\hline 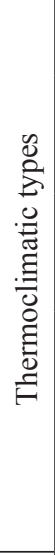 & $\Leftrightarrow$ & 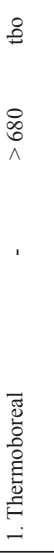 & 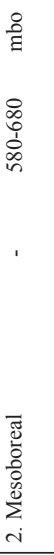 & 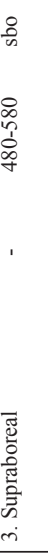 & 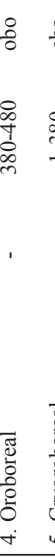 & 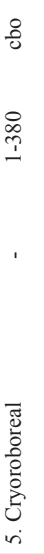 & 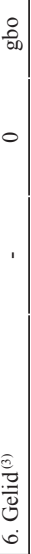 & 荌 & 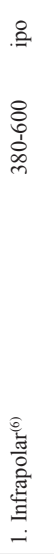 & 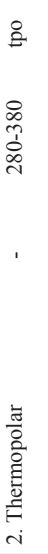 & 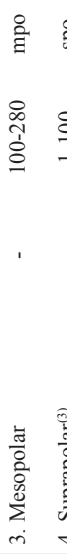 & $\begin{array}{l}\frac{8}{1} \\
.\end{array}$ & 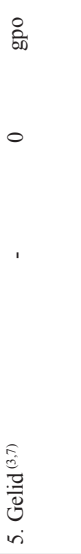 & 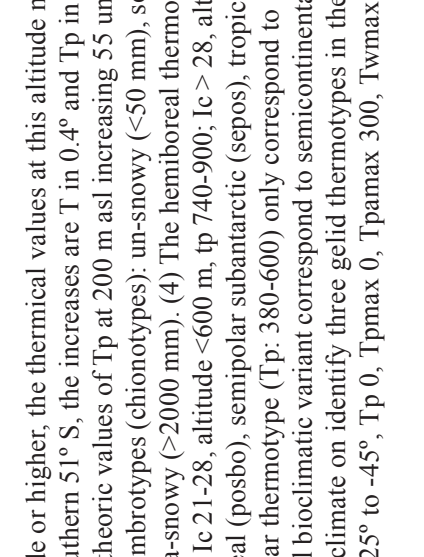 \\
\hline 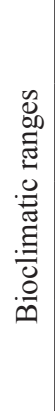 & $\begin{array}{l}\Leftrightarrow \\
\Leftrightarrow \\
\therefore\end{array}$ & 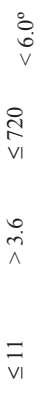 & $\begin{array}{l}\stackrel{0}{\wedge} \\
\stackrel{\sim}{\wedge} \\
\stackrel{\vec{I}}{=}\end{array}$ & $\begin{array}{l}\stackrel{\infty}{N} \\
\stackrel{\sim}{N}\end{array}$ & $\begin{array}{l}8 \\
\infty \\
\mathrm{c}_{1}\end{array}$ & $\begin{array}{l}\dot{0} \\
\dot{0} \\
\text { VI }\end{array}$ & $\begin{array}{l}e^{\circ} \\
\text { vit }\end{array}$ & @ & $\begin{array}{l}\stackrel{i}{\wedge} \\
= \\
\vec{v} 1\end{array}$ & $\stackrel{\vec{I}}{=}$ & $\begin{array}{ll} & \\
\wedge & \\
0 & \\
\dot{\lambda} & \\
\wedge & \\
\vec{\lambda} & \end{array}$ & 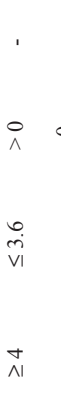 & i & 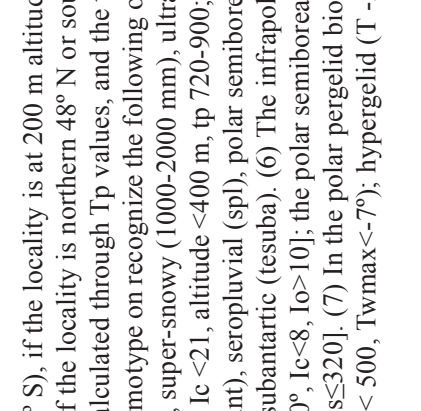 \\
\hline 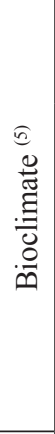 & 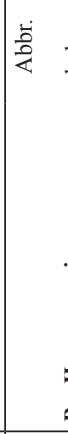 & 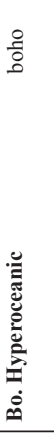 & 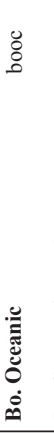 & 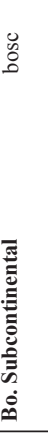 & 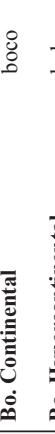 & 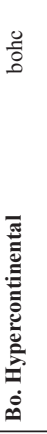 & 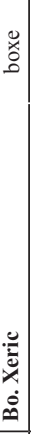 & 营 & 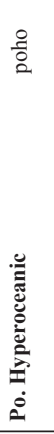 & 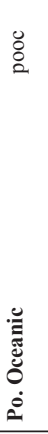 & 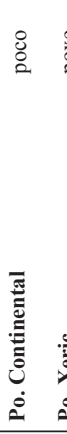 & 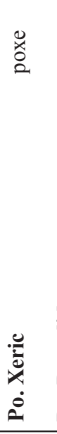 & 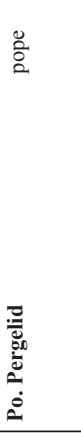 & 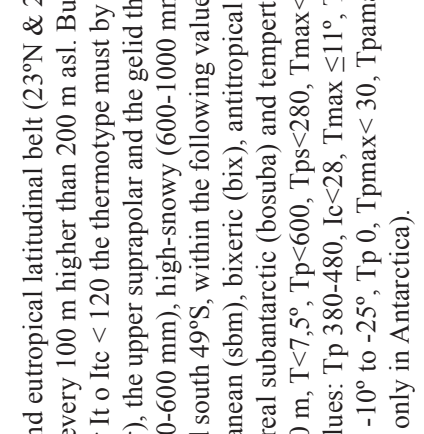 \\
\hline 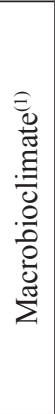 & $\overline{\mathrm{g}}$ & 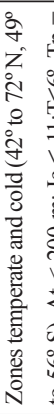 & 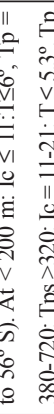 & 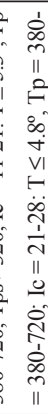 & & 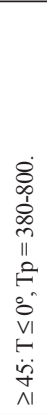 & & & 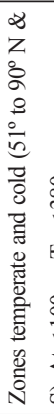 & & & & & 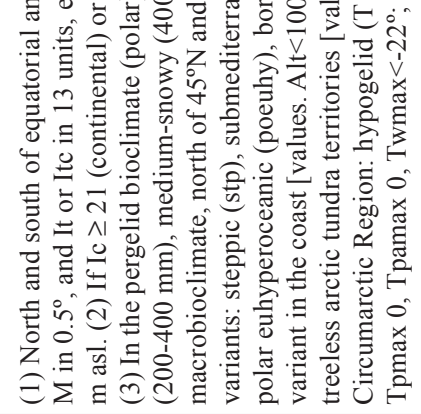 \\
\hline
\end{tabular}




\begin{tabular}{|c|c|c|c|c|c|c|c|c|c|c|c|c|c|c|c|c|c|c|c|c|c|}
\hline 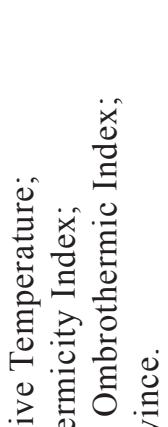 & 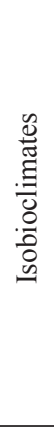 & 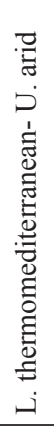 & 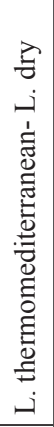 & 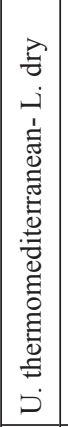 & 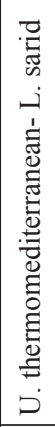 & 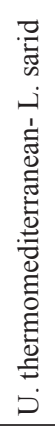 & 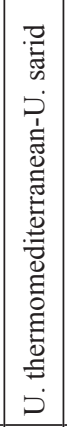 & 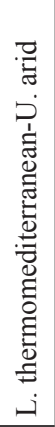 & 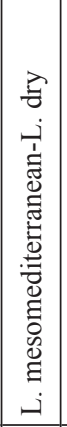 & 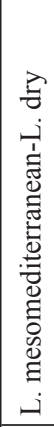 & 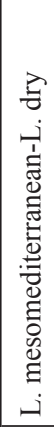 & 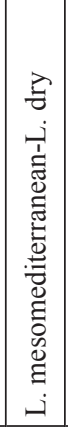 & 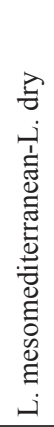 & 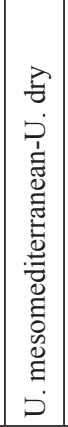 & 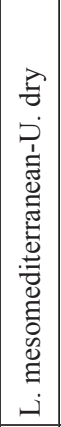 & 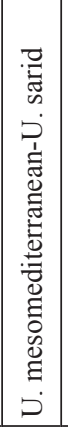 & 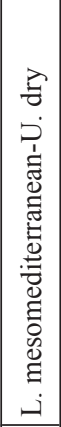 & 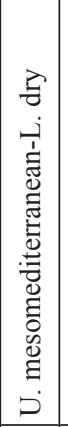 & 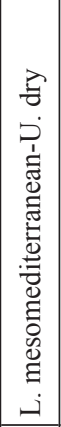 & 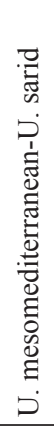 & 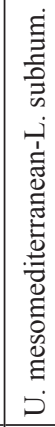 \\
\hline & $\Omega^{\infty}$ & $\stackrel{0}{0}$ & $\stackrel{9}{0}$ & $\stackrel{\infty}{\circ}$ & $\frac{\pi}{0}$ & $\stackrel{1}{0}$ & $\hat{\jmath}$ & $\overrightarrow{\widetilde{o}}$ & : & $\stackrel{+}{0}$ & $\stackrel{\infty}{0}$ & $\stackrel{1}{\circ}$ & $\stackrel{1}{0}$ & $\stackrel{n}{a}$ & กิ & J. & : & $\stackrel{0}{0}$ & $\stackrel{\overbrace{}}{\overbrace{}}$ & $\stackrel{ \pm}{0}$ & $\frac{9}{0}$ \\
\hline 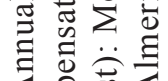 & $\varrho$ & $\stackrel{0}{\circ}$ & $\stackrel{2}{0}$ & $\stackrel{m}{0}$ & $\stackrel{8}{0}$ & $\stackrel{0}{0}$ & $\stackrel{8}{0}$ & $\stackrel{\infty}{0}$ & $\stackrel{\infty}{0}$ & $\stackrel{3}{0}$ & $\stackrel{3}{0}$ & $\stackrel{3}{0}$ & $\stackrel{0}{0}$ & $\stackrel{ \pm}{0}$ & $\stackrel{\circ}{\circ}$ & $=$ & $\stackrel{0}{\circ}$ & $\stackrel{5}{0}$ & $=$ & กี & ڤึ \\
\hline & $\varrho^{\circ}$ & $\stackrel{t}{0}$ & $\stackrel{\circ}{0}$ & $\stackrel{n}{n}$ & $\overline{0}$ & $\hat{n}$ & ñ & $\stackrel{n}{0}$ & $\stackrel{8}{-}$ & $\stackrel{0}{0}$ & $\begin{array}{l}\infty \\
0 \\
0\end{array}$ & $\tilde{0}$ & $\tilde{o}$ & $\stackrel{0}{=}$ & $\bar{a}$ & $\stackrel{\Xi}{=}$ & $\stackrel{\text { ç }}{-}$ & $\vec{\sigma}$ & oे & $\bar{n}$ & م. \\
\hline 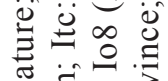 & $\stackrel{\circ}{ }$ & $\hat{o}$ & $\stackrel{\sim}{\sim}$ & $\stackrel{+}{i}$ & $\exists$ & $\exists$ & $\Xi$ & $\stackrel{\infty}{\infty}$ & $\tilde{\sim}$ & $\stackrel{\infty}{i}$ & $\ddot{n}$ & $\hat{i}$ & $\stackrel{\sim}{i}$ & $\stackrel{\vec{r}}{\dot{r}}$ & $\begin{array}{l}\infty \\
i\end{array}$ & 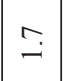 & $\begin{array}{l}\infty \\
i\end{array}$ & $\stackrel{\tilde{i}}{i}$ & $\stackrel{\circ}{\dot{r}}$ & I & $\vec{m}$ \\
\hline 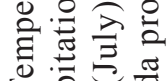 & « & $\stackrel{\text { I }}{=}$ & वे & $\tilde{6}$ & ஜू & $\frac{0}{a}$ & $\stackrel{m}{\sim}$ & $\stackrel{\text { I }}{=}$ & ¿্ర & $\ddot{\infty}$ & 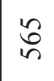 & : & $\stackrel{n}{n}$ & $\frac{\infty}{n}$ & 者 & $\vec{n}$ & $\tilde{n}$ & $\vec{F}$ & if & $\ddot{0}$ & $\tilde{\approx}$ \\
\hline 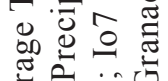 & $\stackrel{0}{0}$ & $\ddot{i}$ & $\stackrel{\stackrel{\circ}{\mathrm{i}}}{\mathrm{I}}$ & $\vec{n}$ & $\ddot{n}$ & $\hat{\sigma}$ & $\stackrel{m}{\infty}$ & గֶ. & $\begin{array}{l}0 \\
\infty \\
-\end{array}$ & $\stackrel{\vec{g}}{\dot{g}}$ & $\stackrel{\circ}{\infty}$ & $\stackrel{\circ}{\stackrel{.}{\Xi}}$ & $\stackrel{\text {. }}{\check{\Xi}}$ & $\stackrel{a}{\dot{b}}$ & $\stackrel{\circ}{\check{I}}$ & $\stackrel{9}{\check{I}}$ & $\stackrel{\infty}{\infty}$ & $\ddot{i}$ & $\stackrel{0}{\dot{\varphi}}$ & $\ddot{\sigma}$ & $\stackrel{\infty}{=}$ \\
\hline & 里 & $\stackrel{\infty}{\mathcal{\gamma}}$ & Эे & ñ & $\underset{\sim}{\stackrel{\infty}{~}}$ & $\underset{\sim}{\stackrel{D}{~}}$ & $\frac{t}{n}$ & $\stackrel{\circ}{\ni}$ & $\cong$ & స్ల & $\stackrel{\infty}{\mathrm{N}}$ & \&े & $\overrightarrow{\widetilde{m}}$ & $\overrightarrow{\mathrm{D}}$ & 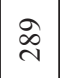 & 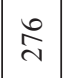 & ळे & $\underset{\sim}{\widetilde{N}}$ & ন্ & $\stackrel{\infty}{\leftrightarrow}$ & मे \\
\hline 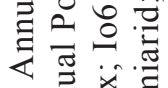 & 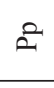 & 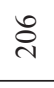 & $\hat{\infty}$ & $\stackrel{゚}{\stackrel{7}{r}}$ & $\underset{\sim}{\stackrel{f}{~}}$ & $\stackrel{\text { I }}{\mathrm{d}}$ & in & $\Xi$ & $\vec{n}$ & d & के & ஓ̊ & $\stackrel{\Xi}{F}$ & $\stackrel{̃}{i n}$ & $\stackrel{\infty}{+}$ & $\underset{\sim}{\stackrel{D}{N}}$ & : & s & $\bar{n}$ & $\stackrel{\infty}{\infty}$ & $\hat{\widehat{\sigma}}$ \\
\hline & $a$ & ๕ٌ & $\hat{\infty}$ & $\stackrel{゚}{\stackrel{7}{+}}$ & $\vec{J}$ & $\stackrel{\sim}{\sim}$ & in & $\Xi$ & 采 & $\stackrel{+}{a}$ & के & $\stackrel{\leftrightarrow}{\circ}$ & $\stackrel{\vartheta}{F}$ & $\underset{i}{\tilde{n}}$ & $\stackrel{\infty}{+}$ & 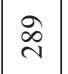 & 官 & $\hat{\delta}$ & $\bar{n}$ & $\stackrel{\infty}{\infty}$ & $\hat{6}$ \\
\hline $\begin{array}{l}\tilde{a} \\
\tilde{\Xi}\end{array}$ & $\Leftrightarrow$ & 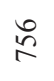 & $\frac{\Delta}{\pi}$ & $\bar{尺}$ & $\stackrel{\text { I }}{I}$ & $\underset{⿱}{\stackrel{N}{r}}$ & $\stackrel{\infty}{\sim}$ & $\underset{n}{*}$ & $\widehat{i}$ & $\vec{f}$ & 2 & $\stackrel{\wp}{r}$ & $\tilde{i}$ & $\tilde{b}$ & $\overline{8}$ & $\vec{b}$ & $\stackrel{\infty}{i}$ & $\underset{ర}{ \pm}$ & త్ర & $\stackrel{Ð}{\gtrless}$ & f \\
\hline & $\approx$ & đั & $\stackrel{\infty}{5}$ & $\stackrel{\infty}{5}$ & $\stackrel{\vec{\sim}}{ }$ & $\stackrel{\bullet}{\sim}$ & $\stackrel{\grave{N}}{\stackrel{\sim}{N}}$ & ठั & d & $\stackrel{2}{\beth}$ & $\stackrel{\infty}{\underset{\infty}{\infty}}$ & $\begin{array}{l}\sqrt{\infty} \\
\infty\end{array}$ & $\stackrel{0}{2}$ & $\begin{array}{l}\infty \\
: \\
0\end{array}$ & $\stackrel{\infty}{\stackrel{\Xi}{I}}$ & $\stackrel{\Xi}{\Xi}$ & $\stackrel{\widetilde{\infty}}{\infty}$ & $\stackrel{\infty}{\stackrel{\Xi}{\Xi}}$ & $\stackrel{n}{\Xi}$ & $\stackrel{0}{I}$ & త్ర \\
\hline & $r$ & $\begin{array}{l}\infty \\
\infty \\
\infty\end{array}$ & $\underset{\infty}{\sim}$ & $\stackrel{n}{\varrho}$ & $\stackrel{i}{\beth}$ & $\begin{array}{l}0 \\
\infty \\
\infty\end{array}$ & $\stackrel{\check{I}}{\check{I}}$ & 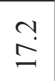 & $\stackrel{m}{n}$ & ị & $\stackrel{\nabla}{a}$ & $\stackrel{n}{n}$ & $\stackrel{0}{\circ}$ & $\stackrel{\vec{\partial}}{\overrightarrow{2}}$ & $\stackrel{\sim}{ \pm}$ & $\begin{array}{l}\infty \\
\dot{\Xi}\end{array}$ & $\stackrel{m}{n}$ & $\stackrel{\stackrel{Y}{ \pm}}{\Xi}$ & $\begin{array}{l}0 \\
\stackrel{+}{J}\end{array}$ & $\stackrel{?}{ \pm}$ & $\begin{array}{l}\stackrel{\circ}{g} \\
\stackrel{\sim}{2}\end{array}$ \\
\hline 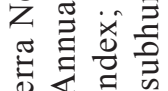 & 劳 & 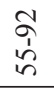 & $\frac{a}{i}$ & $\begin{array}{l}\vec{a} \\
\hat{n}\end{array}$ & ๙ิ่ & $\begin{array}{l}\infty \\
0 \\
n \\
n \\
n\end{array}$ & $\frac{\$}{i}$ & $\begin{array}{l} \pm \\
\dot{0} \\
\dot{0}\end{array}$ & $\frac{9}{1}$ & 啇 & $\frac{2}{6}$ & $\begin{array}{l}\stackrel{a}{1} \\
\hat{n}\end{array}$ & $\begin{array}{l}\text { aे } \\
\text { in }\end{array}$ & $\frac{\tilde{a}}{\dot{i}}$ & $\begin{array}{l}\widetilde{a} \\
\text { aे }\end{array}$ & $\frac{\bar{a}}{a}$ & $\frac{\sigma}{b}$ & $\frac{8}{1}$ & 令 & $\begin{array}{l}\text { aे } \\
\text { bे }\end{array}$ & $\stackrel{\sigma}{\dot{s}}$ \\
\hline 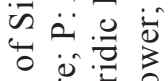 & $\underset{\Xi}{\Xi}$ & $\grave{\cong}$ & 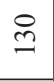 & $\stackrel{8}{7}$ & ষ্ণ & ஓ্ণ & $\frac{0}{6}$ & $\frac{0}{6}$ & ద్రి & $\stackrel{ }{ }$ & ิㅗ & $\stackrel{\sim}{\sim}$ & $\hat{n}$ & $\underset{⿱}{\stackrel{N}{*}}$ & $\stackrel{\circ}{\infty}$ & $\stackrel{\sim}{0}$ & ஓి & $\frac{0}{a}$ & $\vec{\Omega}$ & ฉू & $\stackrel{\circ}{\circ}$ \\
\hline 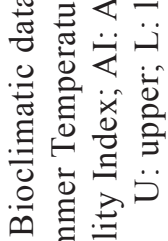 & 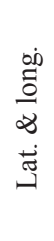 & 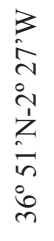 & $\begin{array}{l}3 \\
0 \\
0 \\
0 \\
i n \\
i \\
0 \\
0 \\
0 \\
0 \\
0\end{array}$ & $\begin{array}{l}3 \\
i \\
i \\
0 \\
i \\
i \\
\dot{1} \\
0 \\
0 \\
0 \\
n\end{array}$ & $\begin{array}{l}3 \\
i \\
\infty \\
\vdots \\
i \\
i_{0} \\
\infty \\
0 \\
i \\
0\end{array}$ & $\begin{array}{l}3 \\
i \\
i \\
\vdots \\
i \\
i \\
i \\
o \\
\vdots \\
m\end{array}$ & $\begin{array}{l}3 \\
i \\
i \\
0 \\
i \\
i \\
\dot{1} \\
0 \\
0 \\
0 \\
n\end{array}$ & $\begin{array}{l}3 \\
0 \\
0 \\
\vdots \\
\vdots \\
i \\
i \\
0 \\
0 \\
0 \\
0\end{array}$ & $\begin{array}{l}3 \\
0 \\
0 \\
0 \\
0 \\
1 \\
i \\
0 \\
0 \\
0 \\
0 \\
0\end{array}$ & $\begin{array}{l}3 \\
3 \\
i \\
0 \\
i \\
i \\
i n \\
i n \\
0 \\
0 \\
m\end{array}$ & $\begin{array}{l}3 \\
0 \\
0 \\
0 \\
0 \\
1 \\
z \\
1 \\
1 \\
0 \\
0 \\
m\end{array}$ & 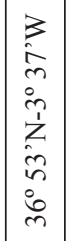 & $\begin{array}{l}3 \\
i \\
\infty \\
i \\
i \\
i \\
-1 \\
i \\
m\end{array}$ & $\begin{array}{l}3 \\
0 \\
0 \\
0 \\
0 \\
i \\
z \\
a \\
0 \\
0 \\
m\end{array}$ & $\begin{array}{l}3 \\
i \\
i \\
o \\
i n \\
i \\
\infty \\
\infty \\
\infty \\
\infty \\
m\end{array}$ & $\begin{array}{l}z \\
\vdots \\
0 \\
0 \\
0 \\
z \\
z \\
\vec{n} \\
\vdots \\
0 \\
m\end{array}$ & $\begin{array}{l}3 \\
m \\
m \\
0 \\
i \\
i \\
0 \\
0 \\
0 \\
0\end{array}$ & $\begin{array}{l}3 \\
0 \\
0 \\
0 \\
i \\
i \\
i \\
i \\
0 \\
0 \\
n\end{array}$ & $\begin{array}{l}3 \\
i \\
i n \\
0 \\
0 \\
i \\
i \\
i \\
i n \\
i n \\
0 \\
n \\
n\end{array}$ & $\begin{array}{l}3 \\
0 \\
0 \\
0 \\
\vdots \\
1 \\
i \\
0 \\
0 \\
0 \\
0\end{array}$ & $\begin{array}{l}3 \\
i \\
i \\
0 \\
i \\
i \\
\dot{0} \\
0 \\
\text { c }\end{array}$ \\
\hline 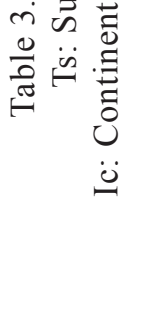 & : & 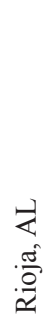 & 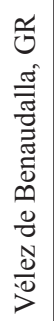 & 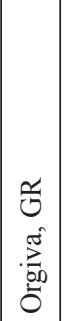 & 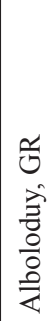 & 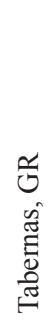 & 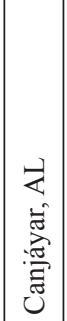 & 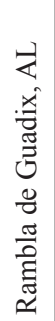 & 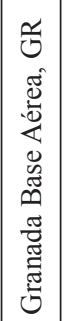 & 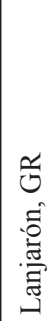 & 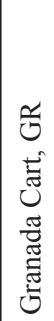 & 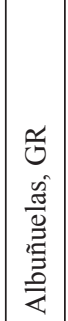 & 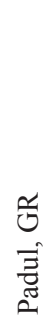 & 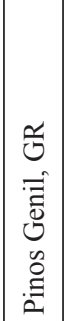 & 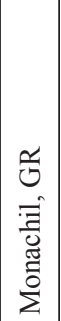 & 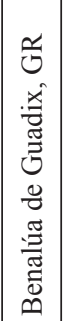 & 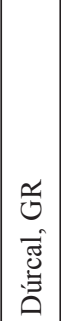 & 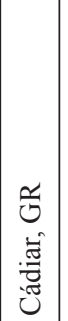 & 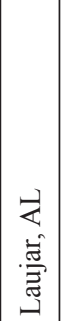 & 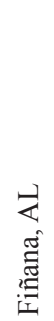 & 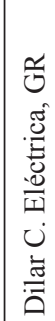 \\
\hline
\end{tabular}




\begin{tabular}{|c|c|c|c|c|c|c|c|c|c|c|c|c|c|c|}
\hline 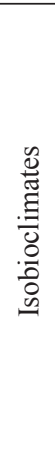 & 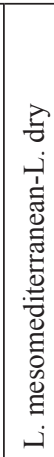 & 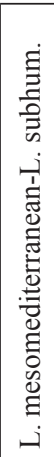 & 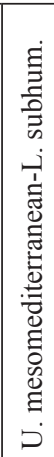 & 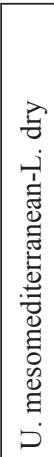 & 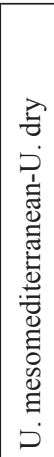 & 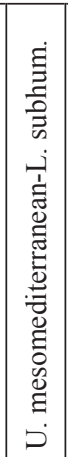 & 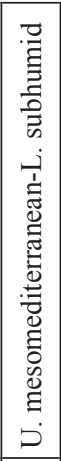 & 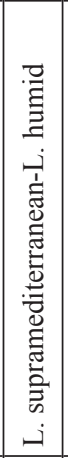 & 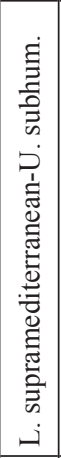 & 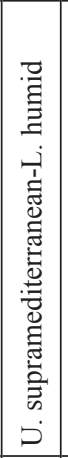 & 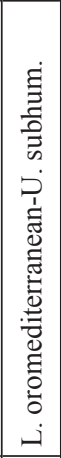 & 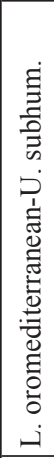 & 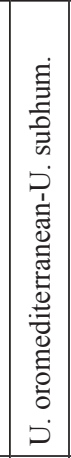 & . \\
\hline$\Omega^{\infty}$ & స్ & ஸุ & iి & & $\infty$ & $\stackrel{\text { I. }}{0}$ & ָิ & $\stackrel{\infty}{\stackrel{\infty}{0}}$ & $\begin{array}{l}\vec{b} \\
0\end{array}$ & $\stackrel{0}{0}$ & กิ & $\stackrel{8}{\circ}$ & nn & \\
\hline 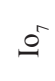 & $\stackrel{8}{0}$ & $\stackrel{7}{0}$ & $\stackrel{9}{0}$ & 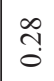 & ֻి & สิ & $\stackrel{5}{0}$ & $\stackrel{ \pm}{0}$ & f. & $\stackrel{0}{0}$ & $\hat{ָ}$ & $\stackrel{m}{0}$ & f. & \\
\hline$\varrho^{\circ}$ & $\stackrel{\infty}{n}$ & $\stackrel{n}{n}$ & 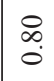 & \&. & $\hat{o}$ & ô & $\stackrel{\overbrace{}}{\Im}$ & $\underset{0}{\sharp}$ & $\stackrel{?}{-}$ & $\stackrel{\sim}{\stackrel{n}{=}}$ & $\begin{array}{l}n \\
n \\
n\end{array}$ & $\stackrel{m}{m}$ & $\stackrel{?}{\stackrel{f}{+}}$ & \\
\hline$\stackrel{\circ}{1}$ & 2 & 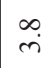 & 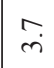 & & $\dot{m}$ & & $\stackrel{\bullet}{+}$ & $\overrightarrow{6}$ & ֶָ. & gे & $\hat{n}$ & in & î & \\
\hline ষ & $\tilde{b}$ & 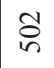 & $\vec{\sim}$ & in & $\bar{n}$ & $\stackrel{0}{+}$ & $\stackrel{\triangleright}{\stackrel{\infty}{+}}$ & $\stackrel{ \pm}{\sim}$ & 商 & 孛 & $\mid \begin{array}{l}\infty \\
\wp\end{array}$ & $\stackrel{m}{m}$ & 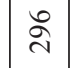 & \\
\hline 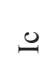 & 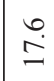 & $\stackrel{\partial}{ \pm}$ & $\stackrel{\overbrace{}}{\stackrel{2}{I}}$ & & $\stackrel{\Upsilon ָ}{\Xi}$ & ? & $\hat{\sigma}$ & $\begin{array}{l}\stackrel{0}{ } \\
\ddot{n}\end{array}$ & $\stackrel{\circ}{\dot{0}}$ & $\stackrel{\circ}{\stackrel{5}{\leftrightarrows}}$ & $\stackrel{n}{\infty}$ & $\stackrel{\infty}{\stackrel{\infty}{\leftrightarrows}}$ & & \\
\hline 巴ֶ & ন & ర్లి & $\tilde{n}$ & & $\overrightarrow{\widetilde{N}}$ & ڤे & $\stackrel{\widetilde{\sim}}{\tilde{n}}$ & $\Xi$ & $\stackrel{\infty}{\mathscr{D}}$ & $\widehat{\cong}$ & 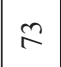 & 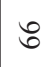 & $\hat{\imath}$ & \\
\hline$\approx$ & $\stackrel{n}{\infty}$ & : & $\bar{n}$ & 슬 & $\ddot{\gamma}$ & $\tilde{\delta}$ & $\tilde{\infty}$ & $\stackrel{\curvearrowright}{\curvearrowright}$ & $\overrightarrow{8}$ & है & $\hat{\sigma}$ & $\hat{n}$ & i & \\
\hline$a$ & $\stackrel{\infty}{\infty}$ & 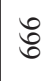 & $\overline{\text { in }}$ & ה & $\stackrel{\tilde{y}}{ }$ & है & $\mathscr{\infty}$ & $\stackrel{\Omega}{\Omega}$ & $\overrightarrow{8}$ & 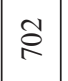 & $\frac{0}{n}$ & $\frac{a}{n}$ & $\overrightarrow{0}$ & \\
\hline$\oplus$ & 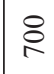 & $\hat{\tilde{b}}$ & $\bar{n}$ & & $\frac{\nabla}{\sigma}$ & ठે & $\frac{m}{6}$ & : & हे & ò & ğ & $\hat{n}$ & & \\
\hline$\Leftrightarrow$ & $\stackrel{ \pm}{\stackrel{2}{\Xi}}$ & క & $\begin{array}{l}\circ \\
\stackrel{2}{2}\end{array}$ & & & $\begin{array}{l}\mathscr{\infty} \\
\stackrel{n}{n}\end{array}$ & 守 & 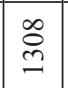 & 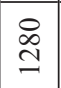 & ๙ิ & $\stackrel{\alpha}{\infty}$ & $\stackrel{\infty}{\infty}$ & nิ & \\
\hline$r$ & & 5 & $\stackrel{m}{\rightarrow}$ & & $\stackrel{\mathfrak{I}}{\mathrm{I}}$ & & $\stackrel{n}{i}$ & $\stackrel{\circ}{\varrho}$ & @્ & $n$ & $\stackrel{n}{r}$ & ? & $\stackrel{\partial}{\dot{\gamma}}$ & \\
\hline 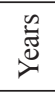 & $\begin{array}{l}\text { के } \\
\text { हे }\end{array}$ & $\frac{\alpha}{\hat{\sigma}}$ & के & & & $\frac{9}{6}$ & $\frac{\sigma}{\dot{a}}$ & & & $\begin{array}{l}\hat{1} \\
\hat{1}\end{array}$ & $\begin{array}{l} \pm \\
\infty \\
\infty\end{array}$ & $\begin{array}{l} \pm \\
\stackrel{1}{0}\end{array}$ & $\frac{\Delta}{n}$ & \\
\hline $\begin{array}{l}\text { 表 } \\
\text { 至 }\end{array}$ & 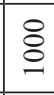 & 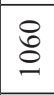 & $\overrightarrow{\widehat{0}}$ & તิ & $\stackrel{\infty}{\stackrel{\infty}{\Xi}}$ & $\begin{array}{l}\stackrel{\infty}{\sim} \\
\stackrel{(1)}{*}\end{array}$ & $\frac{\partial}{m}$ & $\stackrel{\sim}{\cong}$ & $\stackrel{\infty}{\stackrel{\infty}{I}}$ & $\underset{\infty}{\stackrel{f}{-}}$ & $\frac{n}{2}$ & 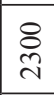 & $\stackrel{\circ}{\stackrel{2}{n}}$ & \\
\hline 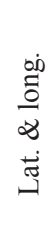 & 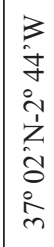 & 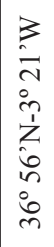 & $\begin{array}{l}3 \\
i n \\
i n \\
o n \\
i \\
i \\
i n \\
i n \\
0 \\
i n\end{array}$ & $\begin{array}{l}3 \\
a \\
\text { in } \\
1 \\
z \\
= \\
\vdots \\
0 \\
m\end{array}$ & 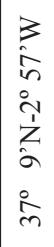 & $\begin{array}{l}3 \\
i \\
\tilde{n} \\
\grave{i} \\
\dot{z} \\
= \\
i \\
i\end{array}$ & 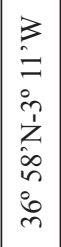 & $\begin{array}{l}3 \\
i \\
i \\
i \\
i \\
i \\
i \\
i n \\
0 \\
0 \\
\text { r. }\end{array}$ & 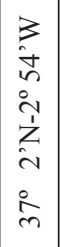 & 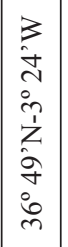 & $\begin{array}{l}3 \\
i n \\
i \\
0 \\
i \\
i \\
a \\
0 \\
i \\
m\end{array}$ & 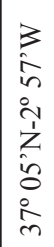 & 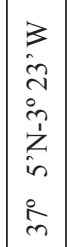 & \\
\hline 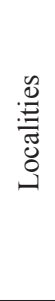 & 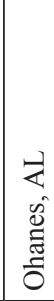 & 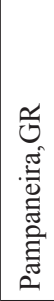 & 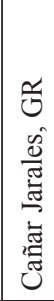 & 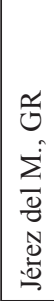 & 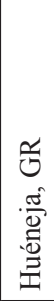 & 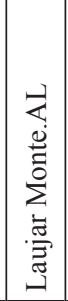 & 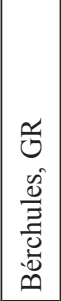 & 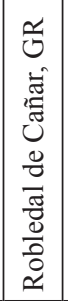 & 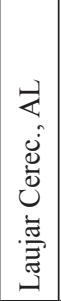 & 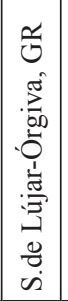 & 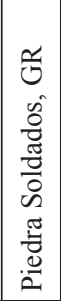 & 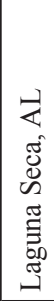 & 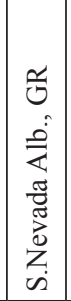 & 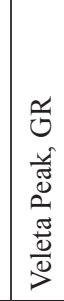 \\
\hline
\end{tabular}


The biogeographic typology proposed to Sierra Nevada National Park is based mostly on previous geobotanic publications of flora (Molero Mesa \& Pérez Raya, 1987; Rivas-Martínez \& al., 1991; Molero Mesa \& González-Tejero, 1996; Fernández Calzado \& Molero Mesa, 2011b; Lorite, 2016; Marfil, 2017 in press.), vegetation, bioclimatology and biogeography, particularly in its correspondence with vegetation series and geoseries of the territory (Rivas Martínez \& al., 1997; Molero Mesa \& Marfil, 2015, 2017; Marfil, 2017 in press.), as well as on other works on vegetation, plant dynamics, and graphic representation of vegetation series, and geoseries in the area (Valle, 1985; Rivas-Martínez \& al., 1986; Molero Mesa \& al., 2001; Losa Quintana \& al., 1986; Lorite \& al., 1997; El Aallali \& al., 1998; Fernández Calzado \& Molero Mesa, 2011a). Also key have been the global studies, vegetation, maps, and the records of the vegetation series of Spain (Rivas-Martínez \& Loidi, 1999; Rivas-Martínez, 1987; Valle \& al., 2003-2005; Rivas-Martínez \& al., 2007, 2011a, 2011b). Geobotanical data have been the essential bases to sketch the new map of the districts and biogeographic territories (Map 1).

\section{Results and Discussion}

\section{Biogeographic districts and territories of Sierra Nevada National Park with their adjacent areas (West Mediterranean Subregion)}

The entire National Park belongs to the Mediterranean Region and has a typical Mediterranean climate, with pronounced summer drought that can last up to 9-12 months, depending on the year, particularly in the southeast low desertic and thermic zones (West Almeria District).

The biogeographic typology recognized and accepted at the district unit rank inside the Na- tional Park includes one region, one subregion, two provinces, five sectors and eight districts (Rivas-Martínez \& al., 2007, 2014, 2017), with two biogeographic territories (countries). Out of the National Park in adjacent areas, there are four well-represented Betic districts: 42e. Filabres Sierran District, 42f. Baza Sierran District, 45b. Alfacar Sierran District and 45d. Almijara Sierran District (Map1):

\section{5c. Trevenque Sierran District}

This district is located between the basins of the Genil, Monachil, Dílar, Durcal, and Torrente rivers, with $196 \mathrm{~km}^{2},(11 \%)$, mostly on dolomites, but also on limestones, calco-dolomites and clays, sometimes vertics. Contact in height with the Nevadense sector and at the base, towards the south, with the sector Alpujarreño-Gadorense. Maximum altitude in the Loma de los Panaderos, 2350 $\mathrm{m}$ asl, Las Sabinas, $2215 \mathrm{~m}$ asl, Trevenque, $2079 \mathrm{~m}$ asl, and Dornajo $2075 \mathrm{~m}$ asl. It spans the lower mesomediterranean to the lower oromediterranean thermotypes, with ombroclimates dry, subhumid, and lower humid at the beginning of oromediterranean belt (2050 $\mathrm{m}$ asl).

This is a diverse, cold district with a large amount of sandy dolomitic, dolomitic, and calco-dolomitic mountains (Trevenque, Tesoro, Dornajo, Sierra del Manar, etc.) where the mesomediterranean barely reaches 1300 $\mathrm{m}$ asl. and the rainfall is particularly high, but with a very permeable substrate. The series of mountain conifers Daphno hispanicaePino nevadensis $S$., in the upper supramediterranean and Rhamno infectoriae-Junipero sabinae $S$. in the lower oromediterranean, are good indicators of the territory, where the Holm oak series represents the largest potential area (Berberido hispanicae-Querco rotundifoliae $S$. and Paeonio coriaceaeQuerco rotundifoliae $S$.). Other bioindicators are included in Table 4. 


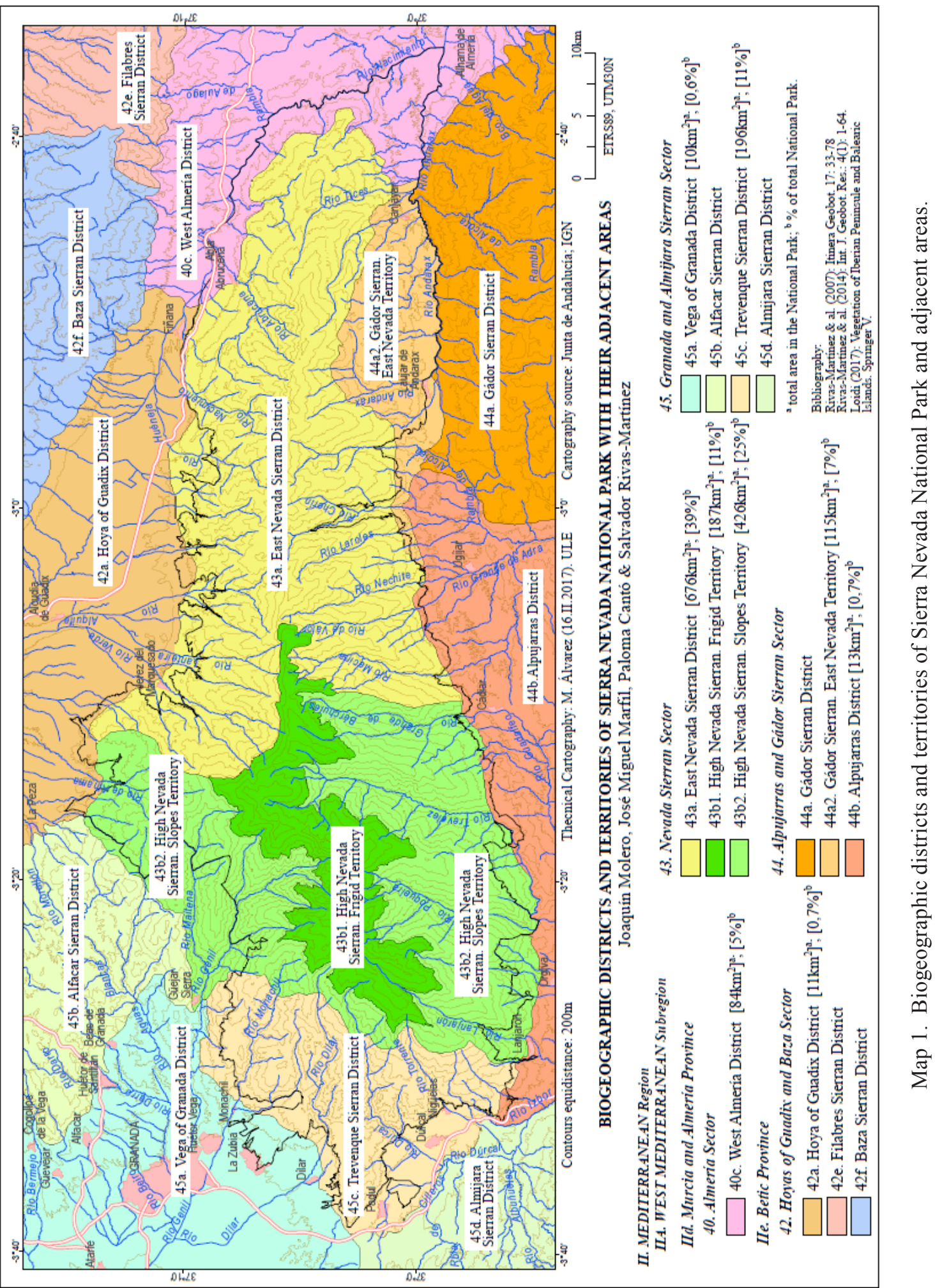


Table 4. Trevenque Sierran District bioindicators. S: sigmetum; T: thermomediterranean;

M: mesomediterranean; S: supramediterranean; O: oromediterranean; C: cryoromediterranean.

\begin{tabular}{|l|c|c|c|c|c|}
\hline \multicolumn{1}{|c|}{ 45c. Trevenque Sierran District. SNNP } & \multicolumn{5}{c|}{ Thermotypes } \\
\hline & T & M & S & O & C \\
\hline Sigmataxa, Syntaxa & & & & & \\
\hline Daphno hispanicae-Pino nevadensis S. & - & - & $\bullet$ & $\bullet$ & - \\
\hline Brachypodio boissieri-Querco rotundifoliae S. & - & $\bullet$ & $\bullet$ & - & - \\
\hline Berberido hispanicae-Querco rotundifoliae S. & - & - & $\bullet$ & - & - \\
\hline Brachypodio boissieri-Pino halepensis S. & - & $\bullet$ & - & - & - \\
\hline Festuco hystricis-Astragaletum granatensis & - & - & - & $\bullet$ & - \\
\hline Convolvulo boissieri-Andryaletum agardhii & - & - & $\bullet$ & - & - \\
\hline Brachypodio boissieri-Trisetetum velutini & - & - & $\bullet$ & - & - \\
\hline Thymo gracilis-Lavanduletum lanatae & - & $\bullet$ & - & - & - \\
\hline Taxa & & & & & \\
\hline Hippocrepis nevadensis & - & - & $\bullet$ & $\bullet$ & - \\
\hline Chamaespartium undulatum & - & - & $\bullet$ & - & - \\
\hline Rothmaleria granatensis & - & - & $\bullet$ & - & - \\
\hline Laserpitium longiradium & - & - & $\bullet$ & - & - \\
\hline Helianthemum pannosum & - & - & $\bullet$ & - & - \\
\hline Erodium boissieri & - & - & $\bullet$ & - & - \\
\hline Lomelosia pulsatilloides & - & - & $\bullet$ & - & - \\
\hline Linaria saturejoides subsp. angustealata & - & $\bullet$ & $\bullet$ & - & - \\
\hline
\end{tabular}

The Nevada Sierran Sector covers $1290 \mathrm{~km}^{2}$, the $75 \%$ of the total area of the park and occupies the central territory, with the highest peaks and metamorphic geological substrata (lastra). Seven large river basins (Guadalfeo, Grande de Adra, Andarax, Nacimiento, Guadix-Guadiana Menor, Genil and Dúrcal) originate in its summits. The thermotypes range from the thermomediterranean to the cryoromediterranean thermic belts, with arid to humid ombrotypes. The plant ecosystems have very high biodiversity.

In the oro- and cryoromediterránean bioclimatic belts, the special climatic conditions, high rate of endemic species and plant communities, afford strong independence to the summits. The melted deep winter snow gives rise to springs, streams, and high-altitude boggy soils, which harbour diverse hygrophilous plant communities (borreguiles). Entire months of winter registering $\mathrm{T}>0^{\circ} \mathrm{C}$ (1 to 6 months) and an aridic summer (Io ${ }_{7-8}<2.0$ ) gives rise to open, desertic-like upper oro- and cryoromediterranean vegetation. The lower oromediterranean belt, which starts at $2100-2150 \mathrm{~m}$ on the northern slope and at $2200-2250 \mathrm{~m}$ on the southern slope, rarely undergoes continuous freezes (gelid months). Before the anthropic destruction of pine forest (Pinus sylvestris subsp. nevadensis) the natural potential vegetation consisted of the dwarf hemispheric juniper communities of Juniperus hemisphaerica, which constituted the first seral vegetation stage of the Sierra Nevada Scots pine forest in the lower horizon. This community of junipers made up the climax vegetation in the medium and upper oromediterranean belts. The Sierra Nevada Scots pine (Pinus sylvestris subsp. nevadensis), widely cultivated, continues to be the potential vegetation particularly on shallow soils in the high levels of the upper supramediterranean horizon, where the woodlands of Quercus pyrenaica and Quercus rotundifolia as well as Acer granatense and Betula fontqueri grow well on deeper soils.

The High Nevada Sierran District (43b) is located in the central-western part of park. It contains all the forest flora and vegetation of the sector and the greatest number of ecosystems, plant communities, and endemic taxa. The marked differences between highlands (upper oro- and cryoromediterranean belts) and the lower areas make it possible to divide the area into two separate territories:

\section{3b1. High Nevada Sierran Frigid Territory}

Is the culminating zone of Sierra Nevada of $\left(187 \mathrm{~km}^{2}, 11 \%\right)$, with a great part of the oromediterranean belt and all the cryoromediterranean belt of the national park. The upper oromediterranean horizon begins at 2500 $\mathrm{m}$ asl (2600 m asl south), with 3-4 months of temperatures below zero degrees and the 
cryoromediterranean from $2800-2850 \mathrm{~m}$ asl (2900-2950 m asl south), with 6 months of temperatures below zero. The series Genisto versicoloris-Junipero hemisphaericae $S$. is the climax dwarf prostrate shrubby juniper in the lower zone, mostly under $2800 \mathrm{~m}$ asl and in the cryoromediterranean a mosaic of permaseries occupy the different habitats of the high mountain. This belt and, in general, most of territory, is a desolate, desert-like environment with a great number of taxa endemic to Sierra Nevada and two types of characteristic ecosystems. The first is xerophytic frigid, which corresponds to associations of Nevadension purpureae and Holcion caespitosi (scree), which are extensive, while the second consists of hygrophilous peaty and boggy communities locally known as borreguiles (Festucion frigidae, Plantaginion nivalis), these being quite, scarce.

The ombrotypes range from the humid at the base south and western part of the territory changing to the dry or even the upper semiarid as the cryoromediterranean belt ascends $(>3000 \mathrm{~m})$. This territory has the highest number of frosty months. Some bioindicators of the territory are included in Table 5.

Table 5. Frigid High Nevada Sierran Summits Territory bioindicators. Ps: permasigmetum, Ms: minorisigmetum; T: thermomediterranean; M: mesomediterranean; S: supramediterranean; $\mathrm{O}$ : oromediterranean; $\mathrm{C}$ : cryoromediterranean.

\begin{tabular}{|l|c|c|c|c|c|}
\hline 43b1. Frigid High Nevada Sierran Summits Territory. SNNP & \multicolumn{5}{|c|}{ Thermotypes } \\
\hline & $\mathrm{T}$ & $\mathrm{M}$ & $\mathrm{S}$ & $\mathrm{O}$ & $\mathrm{C}$ \\
\hline Sigmataxa, Permasigmeta & & & & & \\
\hline Erigeronto frigidi-Festuco clementei Ps. & - & - & - & - & $\bullet$ \\
\hline Genisto versicoloris-Junipero hemisphaericae Ms. & - & - & - & $\bullet$ & - \\
\hline Festuco moleroi-pseudoeskiae Ps. & - & - & - & $\bullet$ & $\bullet$ \\
\hline Violo crassiusculae-Linario glacialis Ps. & - & - & - & $\bullet$ & $\bullet$ \\
\hline Campanulo willkommii-Polysticho lonchitidis Ps. & - & - & - & $\bullet$ & $\bullet$ \\
\hline Ranunculo acetosellifolii-Vaccinio nani Ps. & - & - & - & $\bullet$ & $\bullet$ \\
\hline Nardo strictae-Festuco ibericae Ps. & - & - & - & $\bullet$ & $\bullet$ \\
\hline Saxifrago nevadensis Ps. & - & - & - & - & $\bullet$ \\
\hline Taxa & & & & & \\
\hline Artemisia granatensis & - & - & - & $\bullet$ & $\bullet$ \\
\hline Festuca frigida & - & - & - & $\bullet$ & $\bullet$ \\
\hline Ranunculus alismoides & - & - & - & $\bullet$ & $\bullet$ \\
\hline Trisetum glaciale & - & - & - & $\bullet$ & $\bullet$ \\
\hline Iberis embergeri & - & - & - & $\bullet$ & $\bullet$ \\
\hline Pedicularis verticillata subsp. caespitosa & - & - & - & $\bullet$ & $\bullet$ \\
\hline Vaccinium uliginosum subsp. nanum & - & - & - & $\bullet$ & $\bullet$ \\
\hline Cerastium alpinum subsp. nevadense & - & - & - & - & $\bullet$ \\
\hline
\end{tabular}

\section{3b2. High Nevada Sierran Slopes Territory}

This is a large area of $426 \mathrm{~km}^{2}(25 \%)$ that occupies the siliceous substrates of the high slopes of the entire western Sierra Nevada, located in the Alhama, Genil, Dúrcal, and Guadalfeo (Torrente, Lanjarón, Poqueira, Trevélez) basins. The thermotypes go from the lower mesomediterranean to lower oromediterranean, with dry, subhumid, and lower humid ombroclimates at the beginning of oromediterranean belt. The mesomediterranean bounda- ry is situated around $1200-1300 \mathrm{~m}$ asl in the Genil, Alhama, and Dúrcal basins, increasing towards the south, the Guadalfeo basin, to 1400-1450 $\mathrm{m}$ asl. The anthropogenic climatophilic pine forests (Avenello ibericaePinetum nevadensis) mark the transit to the Frigid Territory and confirm the potentiality of this territory between bioclimatic belts. The oak, Holm oak, ash, maple, birch, and alder forests and other associations typical of high rainfall or humid soils are common. Several bioindicators are listed in Table 6 . 
Table 6. High Nevada Sierran Slopes Territory bioindicators. Gs: geosigmetum; S: sigmetum; T: thermomediterranean; M: mesomediterranean; S: supramediterranean;

O: oromediterranean; C: cryoromediterranean.

\begin{tabular}{|l|c|c|c|c|c|}
\hline \multicolumn{1}{|c|}{ 43b2. High Nevada Sierran Slopes Territory. SNNP } & \multicolumn{5}{c|}{ Thermotypes } \\
\hline Sigmataxa, Syntaxa & T & M & S & O & C \\
\hline Genisto versicoloris-Junipero hemisphaericae S. & & & & & \\
\hline Avenello ibericae-Pino nevadensis S. & - & - & - & $\bullet$ & - \\
\hline Berberido hispanicae-Aceri granatensis S. & - & - & $\bullet$ & $\bullet$ & - \\
\hline Carici camposii-Alno glutinosae Gs. & - & - & $\bullet$ & - & - \\
\hline Aceri granatensis-Fraxino angustifoliae S. & - & - & $\bullet$ & - & - \\
\hline Adenocarpo decorticantis-Querco pyrenaicae S. & - & - & $\bullet$ & - & - \\
\hline Agrostio nevadensis-Genistetum versicoloris & - & $\bullet$ & $\bullet$ & - & - \\
\hline Rhamno cathartici-Loniceretum arboreae & - & - & - & $\bullet$ & - \\
\hline Taxa & - & - & - & $\bullet$ & - \\
\hline Arabis margaritae & & & & & \\
\hline Tephroseris elodes & - & - & - & $\bullet$ & - \\
\hline Thymus pulegioides & - & - & - & $\bullet$ & - \\
\hline Salix caprea & - & - & $\bullet$ & $\bullet$ & - \\
\hline Sorbus hybrida & - & - & $\bullet$ & - & - \\
\hline Heracleum sphondylium subsp. granatense & - & - & $\bullet$ & - & - \\
\hline Laserpitium latifolium subsp. nevadense & - & $\bullet$ & $\bullet$ & - & - \\
\hline Quercus pyrenaica & - & $\bullet$ & $\bullet$ & - & - \\
\hline
\end{tabular}

\section{3a. East Nevada Sierran District}

The eastern district of the sector, with $676 \mathrm{~km}^{2}$ $(39 \%)$, presents a gradual decrease in precipitations towards the east. It is located in the Guadix, Nacimiento, Andarax, and Grande de Adra basins. The high elevation also decreases towards the east: San Juan $2781 \mathrm{~m}$ asl, Chullo $2612 \mathrm{~m}$ asl, Almirez $2519 \mathrm{~m}$ asl, Polarda 2253 $\mathrm{m}$ asl, and Montenegro $1710 \mathrm{~m}$ asl. This district lacks in the cryoromediterranean vegetation belt. The great extent of this territory, with $\mathrm{N}, \mathrm{S}$, and $\mathrm{E}$ exposures, causes appreciable changes in thermotypes, ombrotypes, and continentality. At the summits, the oromediterranean belt begins at $2200 \mathrm{~m}$ asl on the northern slope and at 2300 $\mathrm{m}$ asl on the southern one. The deep soils with large periglacial stones appear to encourage the afforested Pinus sylvestris subsp. nevadensis, at present mostly of anthropogenic character. The seral and climatic vegetation of this area belong to Genisto versicoloris-Junipero hemisphaericae $S$. with Hieracio castellanae-Festucetum longiauriculatae as seral grassland, and particularly at lower altitudes and in the high summits the grasslands of Arenario frigidaeFestucetum indigestae develop quite well.

Thermotypes go from lower mesomediterranean to the lower oromediterranean and the ombrotypes from the semiarid to the lower humid. In the south, less moisture and higher temperatures characterize the transit towards the east. The boundary between the mesoand supramediterranean thermotypes in Rio Grande basin is located at 1450-1500 $\mathrm{m}$ asl and at $1500-1550 \mathrm{~m}$ asl climbing the Andarax river. The semiarid ombrotype begins to predominate towards Montenegro peak. On the northern slopes this boundary lies at $1250-1300 \mathrm{~m}$ asl. It is a cold district, largely semicontinental. It shares with the western district a great number of communities, series, and species. The Quercus pyrenaica oak series is absent, but the Quercus rotundifolia xeric synvariants are significant with respect to the western series. Table 7 shows some bioindicators. 
Table 7. East Nevada Sierran District bioindicators. S: Sigmetum; T: thermomediterranean; M: mesomediterranean; S: supramediterranean; O: oromediterranean; C: cryoromediterranean.

\begin{tabular}{|l|c|c|c|c|c|}
\hline 43a. East Nevada Sierran District. SNNP & \multicolumn{5}{|c|}{ Thermotypes } \\
\hline & $\mathrm{T}$ & $\mathrm{M}$ & $\mathrm{S}$ & $\mathrm{O}$ & $\mathrm{C}$ \\
\hline Sigmataxa, Syntaxa & & & & & \\
\hline Genisto versicoloris-Junipero hemisphaericae S. & - & - & - & $\bullet$ & - \\
\hline Avenello ibericae-Pino nevadensis S. & - & - & $\bullet$ & $\bullet$ & - \\
\hline Dactylido hispanicae-Festucetum scariosae & - & - & $\bullet$ & - & - \\
\hline Adenocarpo decorticantis-Querco rotundifoliae S. & - & $\bullet$ & $\bullet$ & - & - \\
\hline Dorycnio recti-Salici pedicellatae S. & - & $\bullet$ & - & - & - \\
\hline Genisto versicoloris-Cytisetum nevadensis & - & - & - & $\bullet$ & - \\
\hline Teucrio compacti-Quercetum cocciferae & - & $\bullet$ & - & - & - \\
\hline Thymo gracilis-Cistetum ladaniferi & - & $\bullet$ & - & - & - \\
\hline Taxa & - & - & - & - & - \\
\hline Ranunculus girelai & - & - & - & $\bullet$ & - \\
\hline Erysimum baeticum & - & - & $\bullet$ & $\bullet$ & - \\
\hline Artemisia chamaemelifolia & - & - & $\bullet$ & $\bullet$ & - \\
\hline Saxifraga trabutiana & - & - & $\bullet$ & - & - \\
\hline Pinguicula grandiflora & - & - & $\bullet$ & - & - \\
\hline Moehringia fontqueri & - & - & $\bullet$ & - & - \\
\hline Centaurea pulvinata & - & - & $\bullet$ & - & - \\
\hline Sideritis luteola & - & $\bullet$ & - & - & - \\
\hline
\end{tabular}

\section{0c. West Almeria District}

This occupies an area of $83.9 \mathrm{~km}^{2}(4.8 \%)$ in the low basins of the Nacimiento and Andarax rivers in the eastern limits of the National Park and reaches altitudes of close to $800 \mathrm{~m}$ on the slopes of the Sierra Nevada. The geological substrates are marls, sandstones, silts, alluvium materials, and calco-dolomites towards the base of the Sierra de Gádor. The territory has low continentality where it is open towards the Mediterranean. The temperatures are the highest in the park, with thermotype predominant upper thermomediterranean and with little representation of the lower thermomediterranean. Rainfall is scarce, with upper arid and lower and upper semiarid ombrotypes. The vegetation differs markedly from that of the rest of the park, with very little cover and a general absence of trees, very open, poor, desertic, where the shrubby climax vegetation, has practically disappeared and there are some stages with steppe plants. The series, Zizipho loti-Mayteno europaei $S$. is probably extinct in the park. Some of the bioindicators in this district are listed in Table 8.

\section{4a2. Gádor Sierran East Nevada Territory}

Territory with $115 \mathrm{~km}^{2}$ (7\%) in the basin of the Andarax river. Geological substrates sometimes complex, the limestones and calco-dolomites predominate and reach almost $2000 \mathrm{~m}$ asl on the slope of the Buitre. It presents greater oceanity, with upper thermomediterranean to the upper supramediterranean thermotypes, and with the upper semiarid to the upper subhumid ombroclimes. Contact with the Almerian sector means that the two sectors have species in common. In the mesomediterranean dry belt appears the East Penibetic calco-dolomiticolous series of the Holm oak, Paeonio coriaceae-Querco rotundifoliae $S$., synvariant with Phlomis almeriensis whereas in the supramediterranean subhumid belt on similar rocky soils, but higher than $1450 \mathrm{~m}$ asl the synvariant of Echinospartum boissieri is common, with Lavandula lanata belonging to Berberido hispanicae-Querco rotundifoliae $S$. Some bioindicators of this territory are included in Table 9. 
Table 8. West Almería District bioindicators. T: thermomediterranean; M: mesomediterranean; S: supramediterranean; O: oromediterranean; C: cryoromediterranean.

\begin{tabular}{|l|c|c|c|c|c|}
\hline 40c. West Almería District. SNNP & \multicolumn{5}{|c|}{ Thermotypes } \\
\hline & $\mathrm{T}$ & $\mathrm{M}$ & $\mathrm{S}$ & $\mathrm{O}$ & $\mathrm{C}$ \\
\hline Sigmataxa, Syntaxa & & & & & \\
\hline Ephedro fragilis-Pino halepensis S. synv. with Launea lanigera & $\bullet$ & $\bullet$ & - & - & - \\
\hline Zizipho loti-Mayteno europaei S. & $\bullet$ & - & - & - & - \\
\hline Lonicero biflorae-Populo albae S. & $\bullet$ & - & - & - & - \\
\hline Dactylido hispanicae-Lygeetum sparti & $\bullet$ & $\bullet$ & - & - & - \\
\hline Suaedo verae-Salsoletum oppositifoliae & $\bullet$ & $\bullet$ & - & - & - \\
\hline Atriplici glaucae-Hammadetum articulatae & $\bullet$ & $\bullet$ & - & - & - \\
\hline Helianthemo almeriensis-Sideritidetum pusillae & $\bullet$ & - & - & - & - \\
\hline Anabasio hispanicae-Euzomodendretum bourgeani & $\bullet$ & - & - & - & - \\
\hline Taxa & & & & & \\
\hline Hammada articulata & $\bullet$ & $\bullet$ & - & - & - \\
\hline Suaeda pruinosa & $\bullet$ & $\bullet$ & - & - & - \\
\hline Plantago ovata & $\bullet$ & $\bullet$ & - & - & - \\
\hline Whitania frutescens & $\bullet$ & - & - & - & - \\
\hline Euzomodendron bourgaeanum & $\bullet$ & - & - & - & - \\
\hline Anthyllis terniflora & $\bullet$ & - & - & - & - \\
\hline Salsola papillosa & $\bullet$ & - & - & - & - \\
\hline Forsskaolea tenacissima & $\bullet$ & - & - & - & - \\
\hline
\end{tabular}

Table 9. Gádor Sierran East Nevada Territory. T: thermomediterranean; M: mesomediterranean; S: supramediterranean; O: oromediterranean; C: cryoromediterranean.

\begin{tabular}{|l|c|c|c|c|c|}
\hline 44a2. Gádor Sierran East Nevada Territory. SNNP & \multicolumn{5}{|c|}{ Thermotypes } \\
\hline & $\mathrm{T}$ & $\mathrm{M}$ & $\mathrm{S}$ & $\mathrm{O}$ & $\mathrm{C}$ \\
\hline Sigmataxa, syntaxa & & & & & \\
\hline Berberido hispanicae-Querco rotundifoliae S. & - & - & $\bullet$ & - & - \\
\hline Paeonio coriaceae-Querco rot. S. sinv. with Phlomis almeriensis & - & $\bullet$ & - & - & - \\
\hline Rhamno almeriensis-Pino halepensis S. & - & $\bullet$ & - & - & - \\
\hline Helictotricho filifolii-Festucetum scariosae & - & - & $\bullet$ & - & - \\
\hline Convolvulo lanuginosi-Lavanduletum lanatae & - & $\bullet$ & $\bullet$ & - & - \\
\hline Genisto retamoidis-Phlomidion almeriensis & $\bullet$ & $\bullet$ & - & - & - \\
\hline Saturejo micranthae-Thymbrion capitatae & $\bullet$ & $\bullet$ & - & - & - \\
\hline Lapiedro martinezii-Stipetum tenacissimae & $\bullet$ & $\bullet$ & - & - & - \\
\hline Taxa & & & & & \\
\hline Centaurea gadorensis & - & - & $\bullet$ & - & - \\
\hline Teucrium bicoloreum & - & - & $\bullet$ & - & - \\
\hline Echinospartum boissieri & - & $\bullet$ & $\bullet$ & - & - \\
\hline Ephedra nebrodensis & - & $\bullet$ & $\bullet$ & - & - \\
\hline Lavatera oblongifolia & $\bullet$ & $\bullet$ & - & - & - \\
\hline Salvia candelabrum & $\bullet$ & $\bullet$ & - & - & - \\
\hline Phlomis almeriensis & $\bullet$ & $\bullet$ & - & - & - \\
\hline Lavandula lanata & $\bullet$ & $\bullet$ & - & - & - \\
\hline
\end{tabular}


Other small distrital territories exist in Sierra Nevada National Park:

\section{5a. Vega of Granada District}

With $10 \mathrm{~km}^{2}(0.6 \%)$, it includes piedmont and meadows (vega) that converge towards the channel of the river Genil, and has heterogeneous conglomerates in the piedmont with marls and clays in the meadows area.

\section{4b. Alpujarras District}

This extends through the basin of the Guadalfeo river. In the national park, there are only fragments with $13 \mathrm{~km}^{2}(0.7 \%)$. In the lower mesomediterranean dry belt, on calco-dolomitic rocky soils and launas of the Alpujarrid substrata, the natural potential vegetation correspond to a thermic microforest of Quercus rotundifolia that belong to Paeonio coriaceae-Querco rotundifoliae S. with Rhamnus velutina and Phlomis purpurea.

\section{2a. Hoya of Guadix District}

Only marginal inside the Sierra Nevada National Park, this measures $11 \mathrm{~km}^{2}(0.7 \%)$ of disjointed terrain. It has poor representation in the Guadix river basin, with substrates of basic and ultrabasic (mafic) nature. The thermotypes are upper mesomediterranean and lower supramediterranean, with semiarid and dry ombrotypes.

Other adjacent districts appear on the map but not within Sierra Nevada National Park, such as 42e Filabres Sierran D, 42f Baza Sierran D, 45b Alfacar Sierran D, and 54d Almijaran Sierran D.

\section{Conclusion}

A total of 2 provinces, 5 sectors, and 8 districts (one with two territories), map 1, are established in the Sierra Nevada National Park, identified from the application of bioclimatic indices (Rivas-Martínez \& al., 2011c), by delimitation of thermotypes and ombrotypes (bioclimatic belts), the study of the territory and the recognition and discrimination of bioindicators, using flora, plant communities, and vegetation geopermaserie, series, and geoseries. The recognition of homogeneous territories (countries) is an essential tool for the management of Sierra Nevada National Park.

\section{Acknowledgements}

We are grateful for the facilities provided by the management and the PNSN staff, especially J. Sánchez Gutiérrez and Blanca Ramos, for the development of the research carried out. The help of M. Ruiz Girela, J.M. Muñoz Díaz and C. Sánchez Rojas has been important, and especially that of M.R. Fernández Calzado.

\section{Floristic Appendix}

The taxa mentioned in the text follow the nomenclature used in Flora de Andalucía Oriental (Blanca \& al. (Eds.) 2009), except the following

Iberis embergeri Serve: Iberis carnosa Willd. subsp. embergeri (Serve) Moreno

Phlomis almeriensis: Phlomis purpurea L. subsp. almeriensis (Pau) Losa \& Rivas Goday

Ranunculus alismoides Bory: Ranunculus angustifolius DC. subsp. alismoides (Bory) Malag.

Ranunculus girelai: Ranunculus querubicus (J.A. Sánchez Rodr., M.J. Elías \& M.A. Martín) Fern. Prieto, Sanna, M.Pérez \& Cires subsp. girelai Fern.Prieto, Molero Mesa, Muñoz Díaz \& Sanna

Sideritis luteola Font Quer

Vaccinium uliginosum subsp. nanum (Boiss.) Rivas-Martínez, Asensi, Molero Mesa \& F. Valle

\section{References}

Aspizua, R., Barea-Azcón, J.M., Bonet, F.J., Pérez-Luque, A.J. \& Zamora, R. (Eds.) 2012. Observatorio de Cambio Global Sierra Nevada: metodologías de seguimiento. Cons. Med. Amb. Junta de Andalucía.

Anderson, R.S., Jiménez-Moreno, G., Carrión, J.S. \& Pérez Martínez, C. 2011. Postglacial history of alpine vegetation, fire and climate from Laguna de Río Seco, Sierra Nevada, southern Spain. Quaternary Sci. Rev. 30 (13-14): 1615-1629. 
Blanca G., Cabezudo B., Cueto M., Salazar C. \& Morales Torres, C. (Eds.) 2011. Flora Vascular de Andalucía Oriental. 4 vols. Cons. Med. Amb. Junta de Andalucía, Sevilla.

Cantó, P. 2007. Vegetation series as a tool for Biogeography: a case study of the central Iberian Peninsula. Phytocoenologia 37(3-4): 417-442.

Capelo, J.H. 2003. Conceitos e Métodos da Fitossociologia. Formulação Contemporânea e Métodos Numéricos de Análise da Vegeação. Est. Flor. Nal. Soc. Port. Ci. Flor. Oeiras.

Del Arco, M., Acebes, J.R. \&. Pérez de Paz, P.L. 1996. Bioclimatology and climatophilous vegetation of the Island of Hierro (Canary Islands). Phytocoenologia 26(4): 445-479.

Del Arco, M., Salas, M., Acebes, J.R, Marrero, M.C., Reyes Betancort, J.A. \& Pérez de Paz, P.L. 2002. Bioclimatology and climatophilous vegetation of Gran Canaria (Canary Islands). Ann. Bot. Fenn. 39: $15-41$.

El Aallali, A., López Nieto, J.M., Pérez Raya, F. \& Molero Mesa, J. 1998. Estudio de la vegetación forestal en la vertiente sur de Sierra Nevada (Alpujarra Alta granadina). Itinera Geobot. 11: 387-402.

Fernández-Calzado, M.R. 2007. Delimitación del piso crioromediterráneo de Sierra Nevada. Mem. Doc. (inéd). Univ. Granada, 301 pp. + CD.

Fernández-Calzado, M.R. \& Molero Mesa,J. 2011a. The cartography of vegetation in the cryoromediterranean belt of Sierra Nevada: a tool for biodiversity conservation. Lazaroa 32: 101-115.

Fernández-Calzado, M.R. \& Molero Mesa, J. 2011b. High altitude flora of Sierra Nevada (Spain). Flora Medit. 21: 247-259.

Fernández-Calzado, M.R., Molero-Mesa, J., Merzouki, A. \& Casares-Porcel, M. 2012. Vascular plant diversity and climate change in the upper zone of Sierra Nevada, Spain. Plant Biosyst. 146(4): 10441053.

Flörstchütz, F., Menéndez Amor, J. \& Wijmstra, T.A. 1971. Palynology of a thick Quaternary succession in southerm Spain. Paleogeogr. Paleocl. 10: 233-264.

Géhu, J.M. 2006. Dictionnaire de sociologie et synécologie vegetals. Nouvieu-en-Porthieu, Inter-Phytos. Berlin. J. Cramer, 899 pp.

Géhu, J.M. \& Rivas-Martínez, S. 1981. Notions fondamentales de Phytosociologie. In: Dierschke, H. (Ed.). Sytaxonomie. Pp. 5-33. J. Cramer, Vaduz.

Gómez Ortíz, A. \& al. 2002. Mapa geomorfológico de Sierra Nevada. Morfología glaciar y periglaciar. Granada. Consejería de Medio Ambiente. Junta de Andalucía. 86 pp. 1 mapa geomorfológico.

Gómez Ortiz, A., Palacios, D., Palade, B., Vázquez Selem, L. \& Salvador Franch, F. 2012. The deglaciation of the Sierra Nevada (Southern Spain). Geomorphology 159-160: 93-105.

Gottfried, M., Pauli, H., Futschik, A., Akhalkatsi, M., Barancok, P., Benito Alonso, J.L., Coldea, G., Dick, J., Erschbamer, B., Fernandez Calzado, M.R., Kazakis, G., Krajci, J., Larsson, P., Mallaun, M., Michelsen, O., Moiseev, D., Moiseev, P., Molau, U., Merzouki, A., Nagy, L., Nakhutsrishvili, G., Pedersen, B., Pelino, G., Puscas, M., Rossi, G., Stanisci, A., Theurillat, J.P., Tomaselli, M., Villar, L., Vittoz, P., Vogiatzakis, I. \& Grabherr, G. 2012. Continent-wide response of mountain vegetation to climate change. Nat. Clim. Change 2(2): 111-115.

Jiménez-Moreno, G. 2016. Reconstruction of the vegetation from palynological analysis. In: Zamora, R. $\&$ al. (Eds.) Global Change Impacts in Sierra Nevada: Challenges for Conservation. Cons. Med. Amb. Ord. Terr. Junta de Andalucía. 208 pp.

Jiménez-Moreno, G. \& Anderson, R.S. 2012. Holocene vegetation and climate change recorded in alpine bog sediments from the Borreguiles de la Virgen, Sierra Nevada, southern Spain. Quaternary Res. 77 (1): 44-53.

Jiménez-Moreno, G., Garcia Alix, A., Hernández Corbalán, M.D. Anderson, R.S. \& Delgado Huertas, A. 2013. Vegetation, fire, climate and human disturbance history in the southwestern Mediterranean area during the late Holocene. Quaternary Res. 79 (2): 110-122.

Loidi, J. 1991. Vegetation series: its use for small scale geobotanical mapping. Phytocoenosis 3 (n.s.): 119122.

Lorite, J. 2016. An updated checklist of the vascular flora of Sierra Nevada (SE Spain). Phytotaxa 261(1): $1-57$.

Lorite, J., Valle, F. \& Ruiz-Polo, N. 1997. Cartography and vegetation dynamics in the Sierra Nevada, Almería (S.E. Spain). Lagascalia 19(1-2): 601-608.

Lorite, J., Valle, F. \& Salazar, C. 2003. Síntesis de la vegetación edafohigrófila del Parque Natural y Nacional de Sierra Nevada. Monogr. Fl. Veg. Béticas 13: 47-110. 
Lorite, J., Gómez, F., Mota, J.F. \& Valle, F. 2007. Analysis of the orophilous vegetal communities of Baetic ranges in Andalusia (south-eastern Spain): Priority altitudinal-islands for conservation. Phytocoenologia 37: 625-644.

Losa Quintana, J.M., Molero Mesa J. Casares Porcel, M. \& Pérez-Raya, F. 1986. El paisaje vegetal de Sierra Nevada. La cuenca alta del Río Genil. Serv. Publ. Univ. Granada. 285 pp. Granada.

Lousã, M. 2004. Bioclimatologia e series de vegetação de Portugal. Lazaroa 25: 83-86.

Marfil, J.M. 2017. Estudio geobotánico global del Parque Nacional de Sierra Nevada (PNSN). Mem. Doc. (inéd.). Univ. Granada. 202 pp.

Martín, J.M., Braga Alarcón, J.C. \& Gómez Pugnaire M.T. 2008. Itinerarios geológicos por Sierra Nevada. Guía de campo por el Parque Nacional y Parque Natural de Sierra Nevada. Cons. Med. Amb. Junta de Andalucía.

Martínez-Parras, J.M. \& Molero Mesa, J. 1983a. Ecología y fitosociología de Quercus pyrenaica Willd. en la provincia Bética. Los melojares béticos y sus etapas de sustitución. Lazaroa 4: 91-104.

Martínez-Parras, J.M. \& Molero Mesa, J. 1983b. Sobre la alianza Lonicero-Berberidion hispanicae O. de Bolòs 1954, en la parte oriental de la provincia corológica bética. Collect. Bot. (Barcelona) 14: 327-337.

Martínez-Parras, J.M., Peinado, M. \& Alcaraz, F. 1987a. Algunas comunidades orófilas de Andalucía Oriental. Lazaroa 7: 49-53.

Martínez-Parras, J.M., Peinado, M. \& Alcaraz, F. 1987b. Datos sobre la vegetación de Sierra Nevada. Lazaroa 7: 515-533.

Martínez-Parras, J.M., Peinado, M. \& Alcaraz, F. 1987c. Comunidades vegetales de Sierra Nevada (España). Serv. Publ. Univ. Alcalá de Henares. 74 pp.

Mesquita, S., Capelo, J. \& de Sousa, J. 2004. Bioclimatologia da Ilha da Madeira: abordagem numérica. Quercetea 6: 47-59.

Molero Mesa, J. 1999. The vegetation of Sierra Nevada. Itinera Geobot. 13: 105-118.

Molero Mesa, J \& González-Tejero, M.R. 1996. Catálogo y análisis florístico de la Flora orófila de Sierra Nevada. In: Chacón, J. \& Rosúa J.L. (Eds.). $1^{\text {a }}$ Conf. Int. Sierra Nevada, 2 vols. Pp. 271-306. Granada.

Molero Mesa, J. \& Marfil, J.M. 2015. The bioclimates of Sierra Nevada National Park. Int. J. Geobot. Research 5: 1-11.

Molero Mesa, J. \& Marfil, J.M. 2017. Betic and Southwest Andalusia. In: Loidi, J. \& Werger, M.J.A. (Eds.). The Vegetation of the Iberian Peninsula. Springer (in press).

Molero Mesa, J. \& Pérez Raya, F. 1987a. Estudio fitosociológico de los sabinares de Juniperus phoenicea L. en el sector Malacitano-Almijarense (provincia corológica Bética). Lazaroa 7: 301-306.

Molero Mesa, J. \& Pérez Raya, F. 1987b. La flora de Sierra Nevada. Avance sobre el catálogo florístico nevadense. Univ. Granada. 397 pp.

Molero Mesa, J., Pérez Raya, F., López Nieto, J. M., El Aallali, A. \& Hita Fernández, J. A. 2001. Cartografía y evaluación de la vegetación de Sierra Nevada. Mem. Fin. Inv. Cons. Med. Amb. Univ. Granada.

Mota, J.F. \& Valle, F. 1987. Estudio Botánico-Ecológico de las cuencas altas de los ríos Bayárcal, Paterna y Andarax. Serv. Publ. Dip. Almería. 269 pp.

Mota, J.F., Pérez-García, F.J., Jiménez, M.L., Amate, J.J. \& Peñas, J. 2002. Phytogeographical relationships among high mountain areas in the Baetic Ranges (south Spain). Global Ecol. Biogeogr. 2: 497-504.

Oliva, M., Gómez Ortíz, A. \& Schulte, L. 2010. Tendencia a la aridez en Sierra Nevada desde el Holoceno Medio inferida a partir de sedimentos lacustres. Bol. Asoc. Geógr. Esp. (52): 27-42.

Oliva, M., Schulte, L. \& Gómez Ortíz, A. 2011. The role of aridification in constraining the elevation range of Holocene solifluction processes and associated landforms in the periglacial belt of the Sierra Nevada (Southern Spain). Earth Surf. Proc. Land. 36: 1279-1291.

Oliva, M. \& Gómez Ortíz, A. 2012. Late Holocene environ-mental dynamics and climate variability in a Mediterranean high mountain environment (Sierra Nevada, Spain) inferred from lake sediments and historical sources. Holocene 22 (8): 915-927.

Oliva, M., Gómez Ortíz, A., Palacios, D., Salvador Franch, F. \& Salvá, M. 2014. El Cuaternario en el macizo de Sierra Nevada. Evolución paleoambiental y paisaje a partir de la interpretación de registros naturales y documentos de época. Scripta Nova. Rev. Electr. Geogr. Ci. Soc. 18 (472). http://www.ub.es/ geocrit/sn/sn-472.htm.

Pauli, H., Gottfried, M., Dullinger, S., Abdaladze, O., Akhalkatsi, M., Alonso, J.L.B., Coldea, G., Dick, J., Erschbamer, B., Fernández Calzado, M.R., Ghosn, D., Holten, J.I., Kanka, R., Kazakis, G., Kollár, J., Larsson, P., Moiseev, P., Moiseev, D., Molau, U., Molero Mesa, J., Nagy, L., Pelino, G., Puscas, M., Rossi, 
G., Stanisci, A., Syverhuset, A.O., Theurillat, J.P., Tomaselli, M., Unterluggauer, P., Villar, L., Vittoz, P. \& Grabherr, G. 2012. Recent plant diversity changes on Europe's mountain summits. Science 336: 353-355.

Peinado, M., Alcaraz, F. \& Martínez-Parras, J.M. 1992. Vegetation of Southeastern Spain. Flora et Vegetatio Mundi 10: 1-487. J. Cramer, Berlin.

Pérez-Luque, A.J., Pérez-Pérez, R. \& Bonet, F.J. 2016a. Climate change over the last 50 years in Sierra Nevada. In: Zamora, R., Pérez-Luque, A.J., Bonet, F.J., Barea-Azcón, J.M. \& Aspizua, R. (Eds.). Global Change Impacts in Sierra Nevada: Challenges for Conservation. Pp. 24-26. Cons. Med. Amb. Ord. Terr. Junta de Andalucía, Granada.

Pérez-Luque, A.J., Pérez-Pérez, R., Aspizua, R., Muñoz, J.M., \& Bonet, F.J. 2016b. Climate in Sierra Nevada: present and future. In: Zamora, R., Pérez-Luque, A.J., Bonet, F.J., Barea-Azcón, J.M. \& Aspizua, R. (Eds.). Global Change Impacts in Sierra Nevada: Challenges for Conservation. Pp. 27-31. Cons. Med. Amb. Ord. Terr. Junta de Andalucía, Granada.

Pérez Raya, F. 1987. La vegetación en el sector Malacitano-Almijarense de Sierra Nevada: investigaciones sintaxonómicas y sinfitosociológicas. Ser. Publ. Univ. Granada. 350 pp.

Pérez Raya, F. \& Molero Mesa, J. 1988a. Consideraciones sobre el orden Rosmarinetalia Br.- Bl. (1931) 1952 en Sierra Nevada (Granada, España). Mem. Soc. Brot. 28: 137-156.

Pérez Raya, F. \& Molero Mesa, J. 1988b. El orden Festuco hystricis-Poetalia ligulatae en la provincia corológica Bética. Bol. Soc. Brot. ser. 2 63: 147-152.

Pérez Raya, F. \& Molero Mesa, J. 1989. El orden Lygeo spartii-Stipetalia tenacissimae en el sector Malacitano-Almijarense de Sierra Nevada (Granada, España). Ars Pharm. 29(2-3): 245-256.

Pons, A. \& Reille, M. 1988. The Holocene and upper Pleistocene pollen record from Padul (Granada, Spain): a new study. Palaeogeogr. Palaeoclim. 66: 243-263.

Prieto, P. 1971. Vegetación de Sierra Nevada. La cuenca de Monachil. Col. Monogr. Univ. Granada 11. 218 pp.

Quézel, P. 1953. Contribution a l'etude phytosociologique et geobotanique de la Sierra Nevada. Mem. Soc. Brot. 9: 5-82.

Rivas Goday, S. \& Rivas Martínez, S. 1968. Matorrales y tomillares de la Península Ibérica comprendidos en la clase Ononido-Rosmarinetea. Br. Bl.1947. An. Inst. Bot. Cavanilles 25:5-179.

Rivas Goday, S. \& Rivas Martínez, S. 1971. Vegetación potencial de la provincia de Granada. Trab. Dep. Bot. Fis. Veg. 4: 3-85.

Rivas-Martínez, S. 1961. Los pisos de la vegetación de Sierra Nevada. Bol. R. Soc. Esp. Hist. Nat sec. Biol. 59: 55-64.

Rivas-Martínez, S. 1976. Sinfitosociología, una nueva metodología para el estudio del paisaje vegetal. An. Inst. Bot. Cavanilles 33: 179-188.

Rivas-Martínez, S. 1981. Les étages bioclimatiques de la végétation de la Péninsule Ibérique. An. Jard. Bot. Madrid 37(2): 251-268.

Rivas-Martínez, S. 1982a. Mapa de las series de vegetación de la provincia de Madrid. Publ. Serv. For. Med. Ambiente. Dip. Prov. Madrid.

Rivas-Martínez, S. 1982b. Les étages bioclimatiques, secteurs chorologiques et series de végétation de l'Espagne mediterranéenne. Ecol. Medit. 8(1-2): 275-288.

Rivas-Martínez, S. 1983. Series de vegetación de la región eurosiberiana de la Península Ibérica. Lazaroa, 4: $155-166$.

Rivas-Martínez, S. 1984. Pisos bioclimáticos de España. Lazaroa, 5: 33-43.

Rivas-Martínez, S. 1987. Mapa de series de vegetación de España. ICONA Ser Téc. 268 p. +30 mapas. Madrid.

Rivas-Martínez, S. 1988. Bioclimatología, Biogeografía y Series de Vegetación de Andalucía occidental. Lagascalia 15 (extra): 91-119.

Rivas-Martínez, S. 1991. Bioclimatic belts of West Europe (relations between bioclimate and plant ecosystems). Proc. Eur. School Climate Nat. Hazards Course (Arles, 1990). 225-246. Strasbourg.

Rivas-Martínez, S. 1994. Dynamic-zonal phytosociology as landscape science. Phytocoenologia 24: 23-25.

Rivas-Martínez, S. 1996. Geobotánica y Climatología. Discurso investidura Dr. 'Honoris Causa' Universidad de Granada. Serv. Publ. Univ. Granada. 98 p.

Rivas-Martínez, S. 2004. Global Bioclimatics. http://www.globalbioclimatics.org.

Rivas-Martínez, S. 2005a. Avances en Geobotánica. Discurso Apertura Curso Acad. R. Acad. Nal. Farmacia.

Rivas-Martínez, S. 2005b. Notions on dynamic-catenal phytosociology as a basis of landscape science. Plant Biosyst. 139 (2): 135-144. doi: 10.1080/11263500500193790. 
Rivas-Martínez, S. \& Costa, M. 1998. Datos sobre la vegetación y el bioclima del Valle de Arán. Acta Bot. Barcinon. 45: 473-499.

Rivas-Martínez S. \& Loidi, J. 1999. Biogeography of the Iberian Peninsula. Itinera Geobot. 13: 49-68

Rivas-Martínez, S., Fernández-González, F. \& Sánchez-Mata, D. 1986. Datos sobre la vegetación del Sistema central y Sierra Nevada. Op. Bot. Pharm. Compl. 2: 3-136. Madrid.

Rivas-Martínez, S., Asensi, A., Molero-Mesa, J. \& Valle, F. 1991a. Endemismos vasculares de Andalucía. Rivasgodaya 6: 5-76.

Rivas-Martínez, S., Báscones, J.C., Díaz González, T.E., Fernández-González, F. \& Loidi, J. 1991b. La vegetación del Pirineo Occidental y Navarra. Itinera Geobot. 5: 5-456.

Rivas-Martínez, S., Asensi, A., Díez, B., Molero, J. \& Valle, F. 1997. Biogeographical synthesis of Andalusia (southern Spain). J. Biogeogr. 24: 915-928.

Rivas-Martínez, S., Fernández-González, F., Loidi, J., Lousã, M. \& Penas, A. 2001. Syntaxonomical checklist of vascular plant communities of Spain and Portugal to association level. Itinera Geobot. 14: 5-341.

Rivas-Martínez, S., Fernández-González, F., Loidi, J., Lousã, M. \& Penas, A. 2002a. Vascular plant communities of Spain and Portugal. Addenda to the syntaxonomical checklist of 2001. Part I. Itinera Geobot. 15(1): 5-432.

Rivas-Martínez, S., Fernández-González, F., Loidi, J., Lousã, M. \& Penas, A. 2002b. Vascular plant communities of Spain and Portugal. Addenda to the syntaxonomical checklist of 2001. Part II. Itinera Geobot. 15(2): 433-922.

Rivas-Martínez, S. \& al. 2007. Mapas de series, geoseries y geopermaseries de vegetación de España [Memoria del mapa de vegetación potencial de España]. Parte I. Itinera Geobot. 17: 5-436.

Rivas-Martínez, S. \& coautores. 2011a. Mapa de series, geoseries y geopermaseries de vegetación de España, vol. I. Itinera Geobot. 18 (1): 5-424.

Rivas-Martínez, S. \& coautores. 2011b. Mapa de series, geoseries y geopermaseries de vegetación de España, vol. II. Itinera Geobot. 18 (2): 425-800.

Rivas-Martínez, S., Rivas Sáenz, S. \& Penas, A. 2011c. Worldwide bioclimatic classification system. Global Geobot. 1: 1-638 + 4 maps.

Rivas-Martínez, S., Penas, A., Díaz González, T.E., del Río, S. Cantó, P., Herrero, L., Pinto Gomes, C. \& Costa, J.C. 2014. Biogeography of Spain and Portugal. Preliminary typological synopsis. Int. J. Geobot. Research 4(1): 1-64.

Rivas-Martínez, S., Penas, A., Díaz Gozález, T. E., Cantó, P., del Río, S. \& Molero, J. 2017. Biogeographic units of Iberian Peninsula and Balearic islands to district level. In: Loidi, J. \& Werger, M.J.A. (Eds.). The Vegetation of the Iberian Peninsula. Springer (in press).

Salazar, C., Lorite, J., García-Fuentes, A., Torres, J.A., Cano, E. \& Valle, F. 2001. A phytosociological study of the hygrophilous vegetation of Sierra Nevada (Southern Spain). Studia Geobot. 20: 17-32

Sánchez Gómez, P. \& Alcaraz, F. 1993. Flora, Vegetación y Paisaje vegetal de las sierras de Segura orientales. Publ. Inst. Est. Albacetenses. Serie I, Estudios, 69. Albacete.

Sánchez-Gómez, P., Mota, J.F. \& Gómez Mercado, F. 1994. Utilización de criterios bioclimáticos y florísticos en la subdivisión biogeográfica del sector Subbético (provincia Bética). Acta Bot. Malacitana 19: $185-198$.

Valle, F. 1985. Mapa de las series de vegetación de Sierra Nevada. Ecol. Medit. 11: 184-199.

Valle, F. \& al. (Eds.) 2003-2005. Modelos de restauración forestal. Vol. I-IV. Cons. Med. Amb. Junta de Andalucía.

Vera, J.A. (Ed.) 2004. Geología de España. SGE-IGME, Madrid. 890 pp. 2 mapas + CD.

Winkler, M., Lamprecht, A., Steinbauer, K., Hulber, K., Theurillat, J.P., Breiner, F., Choler, P., Ertl, S., Gutierrez Giron, A. Rossi, G., Vittoz, P. Akhalkatsi, M., Bay, C., Benito Alonso, J.L., Bergstrom, T., Carranza, M.L., Corcket, E., Dick, J., Erschbamer, B., Fernandez Calzado, M.R., Fosaa, A.M., G. Gavilan, R., Ghosn, D., Gigauri, K., Huber, D., Kanka, R., Kazakis, G., Klipp, M., Kollar, J., Kudernatsch, T., Larsson, P., Mallaun, M., Michelsen, O., Moiseev, P., Moiseev, D., Molau, U., Molero Mesa, J., Morra di Cella, U., Nagy, L., Petey, M., Puşcaş, M., Rixen, C., Stanisci, A., Suen, M., O. Syverhuset, A., Tomaselli, M., Unterluggauer, P., Ursu, T., Villar, V., Gottfried, M. and Pauli, H. 2016. The rich sides of mountain summits - a pan - European view on aspect preferences of alpine plants. J. Biogeogr. 43(11): 2261-2273.

Zamora, R., Pérez-Luque, A.J., Bonet, F.J., Barea-Azcón, J.M. \& Aspizua, R. (Eds.) 2016. Global Change Impacts in Sierra Nevada: Challenges for Conservation. Cons. Med. Amb. Ord. Terr. Junta de Andalucía, Granada. 208 pp. 
